\title{
Flavor constraints for a vector-like quark of Nelson-Barr type
}

\author{
A.L. Cherchiglia, ${ }^{a}$ G. De Conto ${ }^{a}$ and C.C. Nishi ${ }^{b}$ \\ ${ }^{a}$ Centro de Ciências Naturais e Humanas, Universidade Federal do ABC, \\ Santo André 09.210-170, SP, Brasil \\ ${ }^{b}$ Centro de Matemática, Computação e Cognição, Universidade Federal do ABC, \\ Santo André 09.210-170, SP, Brasil \\ E-mail: adriano.cherchiglia@ufabc.edu.br, george.de.conto@gmail.com, \\ celso.nishi@ufabc.edu.br
}

ABSTRACT: The Nelson-Barr (NB) mechanism to solve the strong CP problem assumes $\mathrm{CP}$ conservation, arranges vanishing $\bar{\theta}$ at tree-level and requires vector-like quarks (VLQs) to transmit the CP breaking to the SM. We analyze the flavor constraints coming from the presence of one such down type VLQ of NB type by performing a global fit on the relevant flavor observables. A comparison is made to the case of one generic VLQ. We find that the allowed parameter space for the VLQ Yukawa couplings and the mixing to the SM are confined to a region much smaller than in the generic case, making the NB case falsifiable in principle.

KEYwords: Beyond Standard Model, CP violation, Quark Masses and SM Parameters

ARXIV EPRINT: 2103.04798 


\section{Contents}

1 Introduction 1

2 Review of the model and parametrization 3

3 Phenomenological constraints for $n_{B}=1 \quad 7$

3.1 VLQ mass

3.2 Constraints from $\left|V_{i j}\right|$

$3.3 \Delta m_{B_{d}}$ and $\Delta m_{B_{s}}$

$3.4 Z \rightarrow b b: R_{b}$

$\begin{array}{lll}3.5 & B_{q} \rightarrow \mu^{+} \mu^{-} & 10\end{array}$

$3.6 \quad B_{d}^{0} \rightarrow J / \psi K_{S} \quad 10$

$3.7 K$ meson system: $\epsilon_{K} \quad 11$

$\begin{array}{lll}3.8 K_{L} \rightarrow \mu \mu & 11\end{array}$

$3.9 K$ meson system: $\epsilon^{\prime} / \epsilon \quad 12$

4 Numerical results $\quad 13$

4.1 Methods 13

$\begin{array}{lll}4.2 & \text { Results } & 14\end{array}$

5 Conclusions $\quad 19$

$\begin{array}{lr}\text { A Inversion formula } & 19\end{array}$

B Approximate formulas for the Yukawas 20

$\begin{array}{ll}\text { C Auxiliary functions } & 21\end{array}$

$\begin{array}{ll}\text { D One up-type NB-VLQ } & 21\end{array}$

\section{Introduction}

The Nelson-Barr (NB) solution [1,2] to the strong CP problem is a very simple mechanism based on the assumption that $\mathrm{CP}$ is a fundamental symmetry of nature $[3,4]^{1}$ - hence the $\mathrm{QCD}$ vacuum angle is zero at tree level - spontaneously broken so that the $\mathrm{CP}$ violation we observe in the SM is reproduced. The mechanism proposes a simple recipe to arrange a vanishing contribution to $\bar{\theta}$ at tree level from quark Yukawa couplings, but still allows large

\footnotetext{
${ }^{1}$ Alternatively, one can assume $\mathrm{P}$ is a fundamental symmetry [5-7]. We will focus on the solution based on $\mathrm{CP}$.
} 
$\mathrm{CP}$ violation to arise in the $\mathrm{SM}$. Then the challenge $[8,9]$ is to keep the calculable radiative corrections to $\bar{\theta}$ to be tiny, $\bar{\theta} \lesssim 10^{-10}$, conforming to the experimental observations [10-13].

A necessary byproduct of the NB mechanism is the presence of vector-like quarks (VLQ) whose mixing with ordinary quarks after spontaneous CP breaking is the only source of $\mathrm{CP}$ breaking that is transmitted to the SM already at tree level. ${ }^{2}$ A simple definition of these VLQs of Nelson-Barr type (NB-VLQs) was given recently for singlet VLQs in ref. [15]. ${ }^{3}$ The simplest complete model was proposed 30 years ago by Bento, Branco and Parada [17], which contained one down-type VLQ and one complex scalar responsible for CP breaking. More recently, ref. [18] improved on this model by imposing a non-conventional CP symmetry which required the addition of two VLQs as the price to protect $\bar{\theta}$ from receiving one-loop corrections. Similarly to these UV completions, the implementation of the NB idea generically leads to theories where CP violation arises only in wave-function renormalization [14]. Flavor alignment may follow if one combines the spontaneous breaking of CP with the breaking of quark family number [19]. See other approaches in refs. [20-30].

However, VLQs appear as byproducts in many other theories, many unrelated to the strong CP problem. Little higgs [31,32] and composite Higgs [33-36] theories comprise a small subset. ${ }^{4}$ Therefore, if VLQs are ever found at the TeV scale, it is pressing that we explore the characteristic features of VLQs related to the strong CP problem and the origin of $\mathrm{CP}$ violation in nature.

Here we study in more detail the case of the presence of a NB-VLQ of charge $-1 / 3$, focusing on the flavor constraints. We perform a global fit on a set of flavor observables and compare the effect of a NB-VLQ with a VLQ of generic type, not necessarily related to the source of CP violation. For one down-type NB-VLQ, ref. [15] has shown that the VLQ couplings to the up type quarks through the charged current necessarily follow a hierarchical pattern, a feature that suppresses the flavor changing effects involving lighter quarks. This nongeneric and hierarchical pattern is interesting if we consider the new physics scales that we can probe by, e.g, $\Delta F=2$ transitions induced by new physics contributions to flavor changing operators of the form

$$
\frac{1}{\Lambda_{i j}^{2}}\left(\bar{d}_{i L} \gamma^{\mu} d_{j_{L}}\right)^{2}
$$

A generic analysis $[37,38]$ at each sector $(i j)=(s d),(b d),(b s)$ leads to the rough bound ${ }^{5}$

$$
\frac{1}{\Lambda_{i j}} \lesssim \frac{\left|V_{t i}^{*} V_{t j}\right|}{3.4 \mathrm{TeV}}
$$

from which follows the hierarchy

$$
\Lambda_{s d}^{-1}: \Lambda_{b d}^{-1}: \Lambda_{b s}^{-1} \sim 0.009: 0.2: 1 .
$$

\footnotetext{
${ }^{2}$ Large CKM CP violation may be also generated at loop level, although it requires strong coupling in the mixing term and supersymmetry to protect $\bar{\theta}[14]$.

${ }^{3}$ A similar setting was proposed some time ago in ref. [16].

${ }^{4}$ Ref. [9] improves the quality of the NB solution by making the spontaneous CP breaking sector composite.

${ }^{5}$ More precise but still generic bounds can be found in the same references.
} 
For one NB-VLQ, this kind of hierarchy is automatically induced. ${ }^{6}$ We will obtain quantitative bounds from the global fit.

We organize this paper as follows: in section 2 we briefly review the model of one NB-VLQ and describe the main features. This includes the seesaw parametrization that was developed previously [15]. In section 3 we list the observables that will be used in the global fit. The methods and the results of the global fit are shown in section 4 . We finally conclude in section 5 .

\section{Review of the model and parametrization}

Our model of one down-type VLQ of Nelson-Barr type (NB-VLQ), denoted by $B_{L, R}$, is defined by the Lagrangian [15]

$$
\begin{aligned}
-\mathscr{L}= & \bar{q}_{i L} \mathscr{Y}_{i j}^{d} H d_{j R}+\bar{q}_{i L} \mathscr{Y}_{i j}^{u} \tilde{H} u_{j R} \\
& +\bar{B}_{L} \mathscr{M}_{j}^{B d} d_{j R}+\bar{B}_{L} \mathscr{M}_{B} B_{R}+\text { h.c. },
\end{aligned}
$$

where $i, j=1,2,3$, and with the additional requirement that $\mathscr{Y}^{u}, \mathscr{Y}^{d}$ are real $3 \times 3$ matrices, $\mathscr{M}_{B}$ is a real mass and only $\mathscr{M}^{B d}$ is a complex row vector. This structure follows from CP conservation and a $\mathbb{Z}_{2}$ symmetry ${ }^{7}$ under which only $B_{L, R}$ are odd, and only $\mathscr{M}^{B d}$ breaks $\mathrm{CP}$ and $\mathbb{Z}_{2}$ softly (spontaneously) realizing the Nelson-Barr mechanism that guarantees $\bar{\theta}=0$ at tree-level $[1,2]$. The SM fields are $q_{i L}, d_{i R}$ and $u_{i R}$, corresponding to the quark doublets, down-type singlets and up-type singlets, respectively. This Lagrangian contains $3\left(\mathscr{Y}^{u}\right)+9\left(\mathscr{Y}^{d}\right)+2\left(\mathscr{M}^{B d}\right)+1\left(\mathscr{M}_{B}\right)=15$ parameters in total (see explicit parametrization below) using the freedom of real orthogonal transformations in the $\mathscr{Y}^{u}$ diagonal basis [15].

In contrast, a generic VLQ is customarily described by the Lagrangian

$$
\begin{aligned}
-\mathscr{L}= & \bar{q}_{i L} Y_{i j}^{d} H d_{j R}+\bar{q}_{i L} Y_{i j}^{u} \tilde{H} u_{j R} \\
& +\bar{q}_{i L} Y_{i}^{B} H B_{R}+\bar{B}_{L} M_{B} B_{R}+\text { h.c. },
\end{aligned}
$$

where $M_{B}$ is expected to be much larger than the electroweak scale. If generic, this lagrangian depends on $3\left(Y^{u}\right)+7\left(Y^{d}\right)+5\left(Y^{B}\right)+1\left(M_{B}\right)=16$ parameters using now the freedom of unitary rotations in $Y^{u}$ diagonal basis. We can see that one more parameter is needed compared to the case of one NB-VLQ. Hence, the NB case is just a subcase and when the lagrangian (2.1) is rewritten in the form (2.2), the various parameters cannot be independent and correlations necessarily appear [15]. In special, only one $\mathrm{CP}$ violating parameter controls all $\mathrm{CP}$ violation in the $\mathrm{NB}$ case while the generic case depends on three CP violating parameters [39-41]. We emphasize this information in table 1.

The changing of basis from (2.1) to (2.2) is easily described by comparing the $4 \times 4$ mass matrix of the down-type quarks following from (2.1) and (2.2), respectively, after EWSB:

$$
\mathrm{NB}: \quad \mathscr{M}^{d+B}=\left(\begin{array}{cc}
\frac{v}{\sqrt{2}} \mathscr{Y}^{d} & 0 \\
\mathscr{M}^{B d} & \mathscr{M}_{B}
\end{array}\right), \quad \text { generic: } \quad M^{d+B}=\left(\begin{array}{cc}
\frac{v}{\sqrt{2}} Y^{d} & \frac{v}{\sqrt{2}} Y^{B} \\
0 & M_{B}
\end{array}\right) .
$$

\footnotetext{
${ }^{6}$ The overall scale, however, is not determined.

${ }^{7} \mathrm{~A}$ larger $\mathbb{Z}_{n}$ or $\mathrm{U}(1)[8]$, a nonabelian global or gauge symmetry [1,2] can be also used. Note that the definition based on $\mathbb{Z}_{2}$ is not sufficient when singlet and doublet VLQs are simultaneously present.
} 


\begin{tabular}{|c|cc|}
\hline & \# of param. & \# of CP odd \\
\hline SM & 10 & 1 \\
One generic VLQ & 16 & 3 \\
One NB-VLQ & 15 & 1 \\
\hline
\end{tabular}

Table 1. Number of parameters in the flavor sector of the SM and with the addition of one VLQ.

Only a unitary transformation from the right is necessary to connect them:

$$
\mathscr{M}^{d+B} W_{R}=M^{d+B} .
$$

The relevant exact relations are given by

$$
\begin{aligned}
M_{B} & =\sqrt{\mathscr{M}^{B d} \mathscr{M}^{B d^{\dagger}}+\mathscr{M}_{B}^{2}}, \\
Y^{B} & =\mathscr{Y}^{d} \mathscr{M}^{B d^{\dagger}} / M_{B}, \\
Y^{d} Y^{d^{\dagger}} & =\mathscr{Y}^{d}\left(\mathbb{1}_{3}-\mathscr{M}^{B d^{\dagger}} \mathscr{M}^{B d} / M_{B}^{2}\right) \mathscr{Y}^{d^{\top}} .
\end{aligned}
$$

For both NB and generic cases, the form $M^{d+B}$ has a simple interpretation in the leading quark seesaw approximation ${ }^{8}$ where $M_{B} \gg v$ : $M_{B}$ is the VLQ mass while $Y^{d} Y^{d^{\dagger}}$ is the leading SM down-type Yukawa matrix squared which contains the CKM CP violation [17]. Therefore, in leading order, the latter is determined from SM input:

$$
Y^{d} Y^{d^{\dagger}}=\frac{2}{v^{2}} V_{d_{L}} \operatorname{diag}\left(m_{d}^{2}, m_{s}^{2}, m_{b}^{2}\right) V_{d_{L}}^{\dagger},
$$

where $V_{d_{L}}$ is the CKM matrix $V_{\mathrm{ckm}}^{\mathrm{sm}}$, except for possible phases from the left. If $V_{d_{L}}=V_{\mathrm{ckm}}^{\mathrm{sm}}$, note that the righthand side of (2.6) contains 7 parameters from the SM down sector. Removing from the counting the three up quark Yukawa couplings in the diagonal basis, we can now concentrate on the 12 parameters in $\mathscr{M}^{d+B}$ for the NB-VLQ case or 13 parameters in $M^{d+B}$ for the generic case; cf. (2.3). We should now concentrate on how to separate the $7 \mathrm{SM}$ input parameters from the BSM parameters. In the generic case, $Y^{d}$ and $Y^{B}$ are independent. Then, in the seesaw approximation, 7 parameters in $Y^{d}$ can be fixed from (2.6), with $V_{d_{L}}=V_{\mathrm{ckm}}^{\mathrm{sm}}$, and the remaining 5 parameters in $Y^{B}$ and one parameter in $M_{B}$ are free BSM parameters. In the NB case, $Y^{d}$ and $Y^{B}$ are not independent and a different parametrization is needed. We now describe the seesaw parametrization devised in ref. [15] for one NB-VLQ. We first choose (2.5a) as one of the parameters instead of $\mathscr{M}_{B}$ and rewrite

$$
\mathscr{M}^{d+B}=\left(\begin{array}{cc}
\frac{v}{\sqrt{2}} \mathscr{Y}^{d} & 0 \\
M_{B} w^{\dagger} & M_{B} \sqrt{1-|w|^{2}}
\end{array}\right) .
$$

Without loss of generality [15],

$$
w=\mathscr{M}^{B d^{\dagger}} / M_{B}=\left(\begin{array}{c}
0 \\
i b \\
a
\end{array}\right),
$$

\footnotetext{
${ }^{8}$ In this approximation, the block diagonalized form of $M^{d+B}$ is simply given by neglecting the $Y^{B}$ term off the diagonal. At this order, $Y^{B}$ will only contribute to the VLQ mixing with the SM but not to the masses.
} 
with $b<a$ and $a^{2}+b^{2}<1$. So $\left\{M_{B}, w\right\}$ contains three parameters while $\mathscr{Y}^{d}$, being real and generic, contains 9 parameters. The combination in the righthand side of $(2.5 \mathrm{c})$ depends on $\mathscr{Y}^{d}$ and $w$, and is partially fixed by SM input in (2.6). However, as is clear from eqs. (2.5b) and (2.5c), both $Y^{d}$ and $Y^{B}$ depends on $\mathscr{Y}^{d}$ and $w$, and we cannot transfer phases from one to another. We then need to consider additional phases ${ }^{9}$ in

$$
V_{d_{L}}=\left(\begin{array}{ccc}
1 & & \\
& e^{i \beta_{1}} & \\
& & e^{i \beta_{2}}
\end{array}\right) V_{\mathrm{ckm}}^{\mathrm{sm}},
$$

in order to take into account SM input in (2.6). Given $\beta_{1}, \beta_{2}$, the real matrix $\mathscr{Y}^{d}$ is partially determined from the inversion formula for (2.5c); see appendix A and ref. [15] for details. The remaining freedom is parametrized by $b$ in $w$ and one additional angle parameter $\gamma$. In this way, in our seesaw parametrization, among the 12 parameters in $\mathscr{M}^{d+B}$, the 7 parameters are fixed from the SM input while the following 5 BSM parameters are free:

$$
\left\{M_{B}, b, \gamma, \beta_{1}, \beta_{2}\right\}
$$

So $\mathscr{Y}^{d}$ and $w$ depend on $b, \gamma, \beta_{1}, \beta_{2}$, and $M_{B}$ can be chosen independently. In special, we restrict

$$
b \in[0.02,1 / \sqrt{2}],
$$

so that the seesaw approximation is valid within $1 \%$ for the mass eigenvalues and the moduli of the $3 \times 3$ block of the CKM matrix [15]. The angle $\gamma$ and phases $\beta_{1}, \beta_{2}$ all vary in the whole range $[0,2 \pi]$. In the generic as well as NB case, the connection with observables are all encoded in the rectangular CKM matrix $V \sim 3 \times 4$,

$$
V=U_{u_{L}}^{\dagger} P U_{d_{L}} \approx V_{d_{L}}\left(\mathbb{1}_{3}-\frac{1}{2} \delta X^{d} \mid \Theta\right)
$$

where $U_{d_{L}}$ is the matrix that diagonalizes either matrix in (2.3) from the left, $P$ is a $3 \times 4$ projection matrix, nonvanishing only for $P_{11}=P_{22}=P_{33}=1$, and

$$
\Theta \equiv V_{d_{L}}^{\dagger} \theta_{L}, \quad \delta X^{d} \equiv \Theta \Theta^{\dagger} .
$$

The diagonalizing matrix of the up sector can be ignored, $U_{u_{L}}=\mathbb{1}_{3}$, in the basis where $Y^{u}=\hat{Y}^{u}$. The expansion parameter in the seesaw is given by

$$
\theta_{L}=\frac{v}{\sqrt{2}} Y^{B} M_{B}^{-1}
$$

The square matrix $X^{d}$ of size 4 that describes the FCNC coupling to $Z$ is

$$
X^{d}=V^{\dagger} V \approx\left(\begin{array}{c|c}
\mathbb{1}_{3}-\delta X^{d} & \Theta \\
\hline * & \Theta^{\dagger} \Theta
\end{array}\right) .
$$

\footnotetext{
${ }^{9}$ In ref. [15], these phases were denoted as $\beta_{2}, \beta_{3}$, respectively.
} 
So we see that all couplings of VLQs to gauge bosons depend solely on the matrix $\Theta$ within the seesaw approximation. For definiteness, we employ the phase convention for $V$ such that

$$
\arg (V)=\left(\begin{array}{cccc}
0 & 0 & * & * \\
\pi & * & 0 & * \\
* & * & 0 & 0
\end{array}\right) .
$$

The stars denote free phases. This convention exhausts all the rephasing freedom and it is easier to implement than the one coming from the angle-phase parametrizations. For the $3 \times 3$ block, this convention is similar to the one employed in ref. [40] and differs slightly only by a tiny phase in the second column. This convention is also very close to the standard parametrization of the $3 \times 3 \mathrm{CKM}$ or to the angle-phase parametrization of the $4 \times 4$ unitary matrix employed in ref. [15]. They differ by tiny phases once SM input is considered. The phase convention (2.16) can be chosen directly for the generic case but it is not automatic for the NB case as the diagonalizing matrix for (2.3) has implicit phase relations. We enforce it after the diagonalization process, a procedure which also redefines $Y^{d}$ and $Y^{B}$ by rephasing from the left. In ref. [15], it was shown that once the SM input was considered, the mixing of the heavy VLQ with the SM quarks followed a hierarchical structure:

$$
\left|V_{u B}\right| \ll\left|V_{c B}\right| \ll\left|V_{t B}\right| .
$$

For a parameter $b$ not so small, the hierarchy roughly follows the hierarchy of $b$-quark mixing:

$$
\left|V_{u B}\right|:\left|V_{c B}\right|:\left|V_{t B}\right| \sim\left|V_{u b}\right|:\left|V_{c b}\right|:\left|V_{t b}\right| \approx 0.004: 0.04: 1,
$$

a feature that is exact in case the VLQ couples exclusively with the third family. The hierarchy also applies to $Y_{i}^{B}$ since they are proportional to $V_{i B}$ at leading order, cf. (2.14). ${ }^{10}$ This hierarchy largely renders the model flavor safe as the most restrictive flavor constraints of flavor changing among the first and second families are naturally suppressed. Here we will test these constraints more quantitatively by performing a global fit. If BSM models are studied in terms of effective field theories and matched onto the SM or effective theories at lower energies, flavor observables can be written in terms of SM parameters and the BSM parameters $Y_{i}^{B}$ and the VLQ mass $M_{B}$. Constraints can then be placed on these BSM parameters. This study was performed in, e.g., ref. [42]. Information on $Y^{B}$ is equivalent to the information on the CKM matrix $V$ and at leading order $Y^{B}$ is proportional to the light-heavy mixing parameters $\theta_{L}$ in (2.14). We can extract $Y^{B}$ independently of the basis for right-handed fields in terms of the mass matrix in (2.3) as

$$
\frac{v}{\sqrt{2}} Y_{i}^{B}=\frac{\left(M^{d+B} M^{d+B^{\dagger}}\right)_{i 4}}{\sqrt{\left(M^{d+B} M^{d+B^{\dagger}}\right)_{44}}} .
$$

This relation is also valid if we replace $M^{d+B}$ by $\mathscr{M}^{d+B}$ from the NB case since the two matrices are related by unitary transformation for right-handed quarks. For the generic

\footnotetext{
${ }^{10}$ See appendix B for an approximate function describing the upper boundary of $\left|Y_{i}^{B}\right|$ as a function of $b$.
} 
case, the mass matrix squared $\left(M^{d+B} M^{d+B^{\dagger}}\right)$ can be reconstructed from the $4 \times 4$ mixing and masses $^{11}$ as

$$
M^{d+B} M^{d+B^{\dagger}}=U_{d_{L}} \operatorname{diag}\left(m_{d}^{2}, m_{s}^{2}, m_{b}^{2}, M_{B}^{2}\right) U_{d_{L}}^{\dagger},
$$

while for the NB case $\mathscr{M}^{d+B}$ itself can be used. The $4 \times 4$ diagonalizing matrix $U_{d_{L}}$ can be parametrized in terms of the standard parametrization for the $3 \times 3$ CKM matrix and additional angles, $\theta_{14}, \theta_{24}, \theta_{34}$, and additional phases $\delta_{1}, \delta_{2}$ [15]; see also refs. [43, 44]. When a specific basis is understood, $Y^{B}$ will denote the Yukawa coupling in the basis where the up-type Yukawas are diagonal $Y^{u}=\hat{Y}^{u}$. However, to compare with ref. [42], we will also need

$$
\tilde{Y}_{i}^{B} \equiv\left(V_{d_{L}}^{\dagger} Y^{B}\right)_{i}
$$

defined in the weak basis where $Y^{d}$ is diagonal when $Y^{B}$ is defined in the basis where $Y^{u}$ is diagonal.

\section{Phenomenological constraints for $n_{B}=1$}

In this section we describe the various observables that will be considered in the fit. Other observables will be predicted from the fit and these are discussed as well.

\subsection{VLQ mass}

Because $V_{i B}$ are hierarchical, the $B$ quark couples dominantly with the top and we can use the current constraint coming from direct searches at the LHC $[45,46]$ :

$$
M_{B} \gtrsim 1.2 \mathrm{TeV} .
$$

Considering the recent analysis [47], we will perform our fit for the conservative value $M_{B}=1.4 \mathrm{TeV}$. Even if $V_{i B}$ are hierarchical and flavor alignment is naturally enforced, we need to test the structure of $V$ against the various flavor observables. The various observables that we will use to constrain the model will be described in the following subsections and is summarized in table 2 . We reserve the letter $B$ for the heavy quark and the $B$-mesons will be always denoted with their subscripts $B_{d}^{0}$ and $B_{s}^{0}$.

\subsection{Constraints from $\left|V_{i j}\right|$}

Most of the moduli $\left|V_{i j}\right|$ of the CKM matrix are extracted from tree level processes. The exceptions are $\left|V_{t d}\right|$ and $\left|V_{t s}\right|$ which are extracted from $B_{d}^{0}$ and $B_{s}^{0}$ meson oscillations through box diagrams involving the top quark. We impose direct constraints on the ones extracted at tree level while the constraints for $\left|V_{t d}\right|$ and $\left|V_{t s}\right|$ are extracted directly from $\Delta m_{B_{d}}$ and $\Delta m_{B_{s}}$ by considering the VLQ contribution; see section 3.3. So these entries may deviate from the SM values. The experimental values for $\left|V_{i j}\right|$ are [48]:

$$
\left|V_{i j}\right|_{\exp }=\left(\begin{array}{ccc}
0.97370(14) & 0.2245(8) & 3.82(0.24) \times 10^{-3} \\
0.221(4) & 0.987(11) & 41.0(1.4) \times 10^{-3} \\
\underline{8.0(0.3) \times 10^{-3}} & \underline{38.8(1.1) \times 10^{-3}} & 1.013(30)
\end{array}\right) .
$$

\footnotetext{
${ }^{11}$ Note that the mass $M_{B}$ here only coincides with the parameter $M_{B}$ in $(2.3)$ in the leading seesaw approximation.
} 
The values for $\left|V_{t d}\right|$ and $\left|V_{t s}\right|$, which are underlined, will not be considered directly. Note that (3.2) shows a somewhat lower value for $\left|V_{u d}\right|$ [48] due to use of updated hadronic uncertainty [49]. These values hint at nonunitarity of the first row of the CKM matrix which can be interpreted in terms of VLQs [50, 51]. For comparison, we can also show the values for the magnitudes of the CKM elements obtained from the combination of the various experiments and assuming unitarity. The result reported by PDG 2020 [48] is

$$
\left|V_{i j}\right|_{\text {exp }}^{\text {fit }}=\left(\begin{array}{ccc}
0.97401(11) & 0.22650(48) & 0.00361_{-0.00009}^{+0.00011} \\
0.22636(48) & 0.97320(11) & 0.04053_{-0.00061}^{+0.00083} \\
0.00854_{-0.00016}^{+0.00023} & 0.03978_{-0.00060}^{+0.00082} & 0.999172_{-0.000035}^{+0.000024}
\end{array}\right) .
$$

The corresponding parameters in the standard parametrization read

$$
\begin{aligned}
s_{12} & =0.22650(48), & s_{13} & =0.00361_{-0.00009}^{+0.00011}, \\
s_{23} & =0.04053_{-0.00061}^{+0.00083}, & \delta & =1.196_{-0.043}^{+0.045},
\end{aligned}
$$

while the Jarlskog invariant is $10^{5} \times J=3.00_{-0.09}^{+0.15}$. In the Wolfenstein parametrization, the same fit leads to

$$
\begin{aligned}
\lambda & =0.22650(48), & A & =0.790_{-0.012}^{+0.017}, \\
\bar{\rho} & =0.141_{-0.017}^{+0.016}, & \bar{\eta} & =0.357(11) .
\end{aligned}
$$

\section{$3.3 \quad \Delta m_{B_{d}}$ and $\Delta m_{B_{s}}$}

The CKM matrix entries $\left|V_{t d}\right|$ and $\left|V_{t s}\right|$ in the SM are currently extracted from $B_{d}^{0}$ and $B_{s}^{0}$ meson oscillations through box diagram contributions. In the presence of VLQs, additional contributions arise due to the exchange of the VLQs. Or, in an EFT description, from the deviation from unitarity of the CKM matrix and FCNC through $Z$ exchange. The $B_{q}^{0}-\bar{B}_{q}^{0}$ mixing for the $q=d, s$ mesons leads to the mass difference

$$
\Delta m_{B_{q}}=2\left|M_{12}^{B_{q}}\right|,
$$

as long-distance effects are negligible. This quantity will be sensitive to

$$
\lambda_{q b}^{t} \equiv V_{t q}^{*} V_{t b} .
$$

The mixing in the presence of a VLQ can be calculated as [52]

$$
M_{12}^{B_{q}}=\frac{G_{F}^{2} m_{W}^{2} f_{B_{q}}^{2} \hat{B}_{B_{q}} m_{B_{q}^{0}}}{12 \pi^{2}}\left[\left(\lambda_{q b}^{t}\right)^{2} \eta_{t t}^{B} S_{0}\left(x_{t}\right)+\Delta_{B_{q}}\right]
$$

where $x_{t} \equiv m_{t}^{2} / m_{W}^{2}$ and

$$
\Delta_{B_{q}}=-8 X_{q b} \lambda_{q b}^{t} \eta_{t t}^{B} Y_{0}\left(x_{t}\right)+\frac{4 \pi s_{w}^{2}}{\alpha_{e}} \eta_{Z}^{B} X_{q b}^{2}
$$

is the VLQ contribution depending on $X_{q b}$. We only retain the dominant top contribution in the box diagrams. The dominant theoretical error comes from the combination $f_{B_{q}} \sqrt{B_{B_{q}}}$ of the meson decay constant and bag parameter. Lattice calculations with three flavors report the average values $f_{B_{d}} \sqrt{B_{B_{d}}}=225(9) \mathrm{MeV}$ and $f_{B_{s}} \sqrt{B_{B_{s}}}=274(8) \mathrm{MeV}$ [53]. Other input parameters are shown in table 3. NLO QCD corrections from running from $m_{W}$ to $m_{b}$ is taken into account by the factors [54] $\eta_{t t}^{B}=0.55, \eta_{Z}^{B}=0.57$, where we neglect the small errors. $S_{0}$ and $Y_{0}$ are Inami-Lim functions given in appendix C. 


\begin{tabular}{|c|c|c|}
\hline Observable $\mathcal{O}_{i}$ & Experimental & SM (c.v [95\%] CL) \\
\hline$\left|V_{i j}\right|$ & Eq. $(3.2)^{a}$ & - \\
\hline$\Delta m_{B_{d}}\left[\mathrm{ps}^{-1}\right]$ & $0.5065(19)[48]$ & $0.5060[0.5031,0.5104]$ \\
\hline$\Delta m_{B_{s}}\left[\mathrm{ps}^{-1}\right]$ & $17.749(21)[48]$ & $17.747[17.711,17.787]$ \\
\hline$B r\left(B_{s} \rightarrow \mu^{+} \mu^{-}\right)$ & $3.0(4) \times 10^{-9}[48]$ & $(3.40[3.13,3.67]) \times 10^{-9}$ \\
\hline$\left|\epsilon_{K}\right|$ & $2.228(11) \times 10^{-3}[48]$ & $(2.227[2.206,2.248]) \times 10^{-3}$ \\
\hline$S_{\psi K_{S}}\left[\sin 2 \beta_{d}\right]$ & $0.699(17)[48]$ & $0.71[0.68,0.74]$ \\
\hline$R_{b}$ & $0.21629(66)[70]$ & $0.21582(11)[71]$ \\
\hline$\left(\epsilon^{\prime} / \epsilon\right)_{\mathrm{NP}}$ & {$[-4,10] \times 10^{-4}[67]$} & - \\
\hline$B r\left(K_{L} \rightarrow \mu^{+} \mu^{-}\right)_{\mathrm{SD}}$ & $<2.5 \times 10^{-9}[60]$ & $(0.86[0.76,0.95]) \times 10^{-9}$ \\
\hline
\end{tabular}

${ }^{a}$ We exclude $\left|V_{t d}\right|,\left|V_{t s}\right|$, symmetrize the errors and inflate them in $50 \%$; see text.

Table 2. Observables considered in the fit. The last column is taken from our fit, except for $R_{b}$, which is not considered in the SM fit.

\section{$3.4 \quad Z \rightarrow b b: R_{b}$}

Given the mixing among the SM $d$-type quarks and the VLQ, not only FCNC will be generated but also diagonal couplings of SM $d$-type quarks to the $Z$ boson will be modified. These modifications can be strongly constrained by the ratio $R_{b}$ [55], since this observable shows a good agreement with the SM prediction; see table 2. For definiteness,

$$
R_{b}=\frac{\Gamma(Z \rightarrow b \bar{b})}{\Gamma(Z \rightarrow \text { hadrons })}
$$

The possible deviations appear in the neutral current with d-type quarks

$$
\mathcal{L}_{Z q q}=-\frac{g}{2 c_{w}} \bar{q} \gamma^{\mu}\left(c_{L q} P_{L}+c_{R q} P_{R}\right) b Z_{\mu}
$$

where, in our model,

$$
c_{L q}=c_{L q}^{\mathrm{SM}}+\delta c_{L q}, \quad c_{L q}^{\mathrm{SM}}=-1+\frac{2}{3} s_{w}^{2}, \quad \delta c_{L q}=1-X_{q q}^{d}, \quad c_{R q}=2 / 3 s_{w}^{2} .
$$

Since only small deviations from the SM couplings will be allowed, one can use an approximate formula for $R_{b}$ obtained by considering only first-order deviations from the tree-level formula

$$
\begin{aligned}
R_{b}^{0} & =\frac{c_{L b}^{2}+c_{R b}^{2}}{\sum_{q}\left(c_{L q}^{2}+c_{R q}^{2}\right)}, \\
R_{b} & =R_{b}^{\mathrm{SM}}\left(1+0.5118 \delta c_{L d}+0.5118 \delta c_{L s}-1.8178 \delta c_{L b}\right)
\end{aligned}
$$

where $R_{b}^{S M}$ is given in table 2 , and $\delta c_{L q}$ are defined in eq. (3.12). 


\section{$3.5 \quad B_{q} \rightarrow \mu^{+} \mu^{-}$}

The rare decays $B_{q} \rightarrow \mu^{+} \mu^{-}, q=d, s$, are extremely suppressed within the SM because it is mediated by a FCNC and are helicity suppressed. Because of the purely leptonic final state, it is also very clean theoretically and then sensitive to new physics. The experimental value for $B_{s}$ is given in table 2 and it is of the order of $10^{-9}$. The branching ratio in the presence of a VLQ can be found in the literature [56] and yields

$$
\operatorname{Br}\left(B_{q} \rightarrow \mu^{+} \mu^{-}\right)=\tau_{B_{q}} \frac{G_{F}^{2}}{16 \pi}\left(\frac{\alpha_{e}}{\pi s_{w}^{2}}\right)^{2} f_{B_{q}}^{2} m_{B_{q}^{0}} m_{\mu}^{2} \sqrt{1-\frac{4 m_{\mu}^{2}}{m_{B_{q}}^{2}}}\left|\eta_{Y}^{2}\right|\left|\lambda_{q b}^{t} Y_{0}\left(x_{t}\right)+\Delta_{\mu \mu}^{B}\right|^{2},
$$

where the deviation of the SM is given by

$$
\Delta_{\mu \mu}^{B}=-\frac{\pi s_{w}^{2}}{\alpha_{e}} X_{q b}
$$

The factor $\eta_{Y}=1.0113$ accounts for QCD corrections in a scheme where NLO electroweak corrections can be neglected [57]. The measurement on $\operatorname{Br}\left(B_{d}^{0} \rightarrow \mu^{+} \mu^{-}\right)$still has a large error and will not be considered in the fit. We will instead present predictions for its value based on the fit. Analogously, the constraint coming from $\operatorname{Br}\left(B_{s}^{0} \rightarrow X_{s} \gamma\right)$ will not be considered because it is weaker than $\operatorname{Br}\left(B_{s}^{0} \rightarrow \mu^{+} \mu^{-}\right)[56]$.

\section{$3.6 \quad B_{d}^{0} \rightarrow J / \psi K_{S}$}

The cleanest measurement of the angle $\beta$ of the $d b$ unitarity triangle is given by the $\bar{b} \rightarrow c \bar{c} \bar{s}$ transition in $B_{d}^{0} \rightarrow J / \psi K_{S}$. It is dominated by only one weak phase in the dominant tree level decay and the penguin pollution is also dominated by the same weak phase. In the presence of the VLQ, additional contributions are induced. The measurement of the direct CP violation decay amplitude $S_{\psi K_{S}}$ occurs through the time-dependent CP asymmetry of $B_{d}^{0}$ and $\bar{B}_{d}^{0}$ to the common final state $J / \psi K_{S}$, which is approximately a CP odd state, and can be written as [40]

$$
S_{\psi K_{S}}=\sin \left(2 \beta+2 \theta_{B_{d}}-2 \theta_{K}\right)
$$

where $\beta$ is the angle

$$
\beta \equiv \arg \left(-\frac{V_{c d} V_{c b}^{*}}{V_{t d} V_{t b}^{*}}\right)=-\arg \left(-\frac{\lambda_{d b}^{c}}{\lambda_{d b}^{t}}\right),
$$

while $\theta_{B}$ and $\theta_{K}$ parametrize the possible deviations from the SM of the mixings in the $B$ and $K$ system as

$$
2 \theta_{B_{d}} \equiv \arg \frac{M_{12}^{B_{d}}}{\left(M_{12}^{B_{d}}\right)_{\mathrm{SM}}}, \quad 2 \theta_{K} \equiv \arg \frac{M_{12}^{K}}{\left(M_{12}^{K}\right)_{\mathrm{SM}}} .
$$

The small phase $\epsilon^{\prime} \equiv \arg \left(-V_{u s} V_{c d} V_{u d}^{*} V_{c s}^{*}\right)$ [58], contributing as $-2 \epsilon^{\prime}$ inside the sine in (3.17), has been neglected. 


\section{7 $K$ meson system: $\epsilon_{K}$}

The $K$ meson system was the first to show measurable CP violation in the SM. The value of $\left|\epsilon_{K}\right| \sim 10^{-3}$ quantifies this indirect $\mathrm{CP}$ violation. The short-distance contribution for the mixing amplitude can be calculated in the presence of a VLQ as (see ref. [52] and references therein)

$M_{12}^{K}=\frac{G_{F}^{2} m_{W}^{2} f_{K}^{2} \hat{B}_{K} m_{K^{0}}}{12 \pi^{2}}\left[\left(\lambda_{d s}^{c}\right)^{2} \eta_{c c}^{K} S_{0}\left(x_{c}\right)+\left(\lambda_{d s}^{t}\right)^{2} \eta_{t t}^{K} S_{0}\left(x_{t}\right)+2 \lambda_{d s}^{c} \lambda_{d s}^{t} \eta_{c t}^{K} S_{0}\left(x_{c}, x_{t}\right)+\Delta_{K}\right]$,

where

$$
\Delta_{K}=-8 X_{d s}\left[\lambda_{d s}^{c} \eta_{Z}^{K} Y_{0}\left(x_{c}\right)+\lambda_{d s}^{t} \eta_{t t}^{K} Y_{0}\left(x_{t}\right)\right]+\frac{4 \pi s_{w}^{2}}{\alpha_{e}} \eta_{Z}^{K} X_{d s}^{2}
$$

the last term being the additional contribution from the VLQ. The $\eta_{i j}^{K}$ are QCD correction factors, $\hat{B}_{K}=0.717(24)[48]$ is the bag parameter. The contribution to the CP violating parameter $\epsilon_{K}$ is given by

$$
\epsilon_{K}=\kappa_{\epsilon} e^{i \phi_{\epsilon}} \frac{\operatorname{Im} M_{12}^{K}}{\sqrt{2} \Delta m_{K}},
$$

where $\kappa_{\epsilon} \simeq 0.94(2)$ [59] includes effects of $\Delta s$ operators and corrections of deviation of $\phi_{\epsilon}=43.52(5)^{\circ}[48]$ from $45^{\circ}$. We use [42] $\eta_{c c}^{K}=1.87(76), \eta_{t t}^{K}=0.5765(65), \eta_{c t}^{K}=0.496(47)$ for the QCD correction factors at NNLO. We also use $\eta_{Z}^{K}=0.60$ [52]. The theoretical prediction for $\Delta m_{K}$ has large error in long-distance contributions and will not be considered. The experimental value is considered in (3.22) instead.

\section{$3.8 \quad K_{L} \rightarrow \mu \mu$}

A strong limit on $\lambda_{s d}^{t}$ and $X_{s d}$ comes from the rare decay $K_{L} \rightarrow \mu^{+} \mu^{-}$. Although clean theoretically, the branching ratio $\operatorname{Br}\left(K_{L} \rightarrow \mu^{+} \mu^{-}\right) \sim 10^{-8}$, containing contributions from a dispersive part, $\operatorname{Re}^{2}(A)$, and a absorptive part, $\operatorname{Im}^{2}(A)$, is known to be dominated by the latter, which can be calculated from $\operatorname{Br}\left(K_{L} \rightarrow \gamma \gamma\right)$. The theoretical prediction for the dispersive part contains large errors from long distance contributions. So we consider the simple bound on the short distance (SD) contribution [60]

$$
\operatorname{Br}\left(K_{L} \rightarrow \mu^{+} \mu^{-}\right)_{\mathrm{SD}}<2.5 \times 10^{-9} .
$$

A more precise constraint would come by considering the quantity $\chi_{S D}[60]$ but we limit ourselves to the constraint above. The short distance part can be calculated by relating it to the decay $K^{+} \rightarrow \mu^{+} \nu$ as [52]:

$$
\frac{B r\left(K_{L} \rightarrow \mu^{+} \mu^{-}\right)_{\mathrm{SD}}}{B r\left(K^{+} \rightarrow \mu^{+} \nu\right)}=\frac{\tau_{K_{L}}}{\tau_{K^{+}}} \frac{\alpha_{e}^{2}}{\pi^{2} s_{w}^{4}\left|V_{u s}\right|^{2}}\left[Y_{\mathrm{NL}} \operatorname{Re}\left(\lambda_{s d}^{c}\right)+\eta_{t}^{Y} Y_{0}\left(x_{t}\right) \operatorname{Re}\left(\lambda_{s d}^{t}\right)+\Delta_{K_{L}}\right]
$$

We use the factor $Y_{\mathrm{NL}}=(2.94 \pm 0.28) \times 10^{-4}$ at $\mathrm{NLO}[52,61]$ and the QCD correction $\eta_{t}^{Y}=1.012[52,62]$. Other input values can be found in table 3. The VLQ contribution is $[52]$

$$
\Delta_{K_{L}}=C_{U 2 Z} \operatorname{Re} X_{s d},
$$

where $C_{U 2 Z}=-\pi s_{w}^{2} / \alpha[63]$. 


\begin{tabular}{|c|c|}
\hline$G_{F}\left[\mathrm{GeV}^{-2}\right]$ & $1.1663787(6) \times 10^{-5}[48]$ \\
\hline$s_{w}^{2}\left(m_{Z}\right)(\overline{\mathrm{MS}})$ & $0.23121(4)[48]$ \\
\hline$m_{W}[\mathrm{GeV}]$ & $80.379(12)[48]$ \\
\hline$m_{Z}[\mathrm{GeV}]$ & $91.1876(21)[48]$ \\
\hline$\alpha\left(m_{Z}\right)^{-1}(\overline{\mathrm{MS}})$ & $127.952(9)[48]$ \\
\hline$\alpha_{s}\left(m_{Z}\right)$ & $0.1179(10)[48]$ \\
\hline$m_{t}\left(m_{t}\right)[\mathrm{GeV}]$ & $161.9[77]^{a}$ \\
\hline$m_{c}\left(m_{c}\right)[\mathrm{GeV}]$ & $1.27[48]$ \\
\hline$y_{d}\left(m_{Z}\right)$ & $1.58 \times 10^{-5}[78]$ \\
\hline$y_{s}\left(m_{Z}\right)$ & $3.13 \times 10^{-4}[78]$ \\
\hline$y_{b}\left(m_{Z}\right)$ & $1.639 \times 10^{-2}[78]$ \\
\hline \multicolumn{2}{|c|}{$\mathrm{SM} \mathrm{fit}$} \\
\hline$A$ & $0.820_{-0.014}^{+0.012}$ \\
\hline$\lambda$ & $0.22655_{-0.00066}^{+0.0000}$ \\
\hline$\lambda$ & $3.18_{-0.12}^{+0.10}$ \\
\hline $10^{5} \times J$ &
\end{tabular}

\begin{tabular}{|c|c|}
\hline$m_{B_{d}}[\mathrm{MeV}]$ & $5279.58(17)[48]$ \\
\hline$m_{B_{s}}[\mathrm{MeV}]$ & $5366.88(16)[48]$ \\
\hline$f_{B_{d}} \hat{B}_{B_{d}}^{\frac{1}{2}}[\mathrm{MeV}]$ & $225(9)[48]$ \\
\hline$f_{B_{s}} \hat{B}_{B_{s}}^{\frac{1}{2}}[\mathrm{MeV}]$ & $274(8)[48]$ \\
\hline$\rho\left(f_{B_{d}} \hat{B}_{B_{d}}^{\frac{1}{2}}, f_{B_{s}} \hat{B}_{B_{s}}^{\frac{1}{2}}\right)$ & $0.951[42,76]$ \\
\hline$\tau_{B_{s}}[\mathrm{ps}]$ & $1.515(4)[48]$ \\
\hline$f_{B_{s}}[\mathrm{MeV}]$ & $228.4(3.7)[53]$ \\
\hline$m_{K^{0}}[\mathrm{MeV}]$ & $497.611(13)[48]$ \\
\hline$\Delta m_{K}[\mathrm{MeV}]$ & $3.484(6) \times 10^{-12}[48]$ \\
\hline$f_{\pi}[\mathrm{MeV}]$ & $130.2(1.2)[48]$ \\
\hline$f_{K} / f_{\pi}$ & $1.192(2)[53]$ \\
\hline \multicolumn{2}{|c|}{$\mathrm{SM}$ fit } \\
\hline $\bar{\rho}$ & $0.154_{-0.012}^{+0.009}$ \\
\hline $\bar{\eta}$ & $0.351_{-0.008}^{+0.010}$ \\
\hline
\end{tabular}

${ }^{a}$ We consider QCD corrections up to four loops with $m_{t}^{\text {pole }}=172.4(7) \mathrm{GeV}[48]$ and $\alpha_{s}\left(m_{t}^{\text {pole }}\right)=$ 0.1077 .

Table 3. Input parameters. Some parameters for specific observables are listed in the text. The last two rows list the Wolfenstein parameters from the SM fit.

\section{$3.9 K$ meson system: $\epsilon^{\prime} / \epsilon$}

The value for $\epsilon^{\prime} / \epsilon$ quantifying direct $\mathrm{CP}$ violation in the SM was until recently predicted to be significantly below the current experimental world average from NA48 [64] and $\mathrm{KTeV}$ [65] collaborations:

$$
\left(\epsilon^{\prime} / \epsilon\right)_{\exp }=(16.6 \pm 2.3) \times 10^{-4} ;
$$

see [66] for a summary of the discrepancy before April 2020. However, the recent 2020 calculation of ref. [67] reports

$$
\left(\epsilon^{\prime} / \epsilon\right)_{\mathrm{SM}}^{(9)}=(13.9 \pm 5.2) \times 10^{-4}
$$

which is well compatible with the experimental value. This value takes into account recent lattice calculations of hadronic matrix elements [68], isospin breaking effects [69] which include the effects of the nonet of lowest-lying mesons and NNLO QCD corrections to EW penguin contributions. The compatibility weakens the possibility for new physics (NP) [66] as the source of deviation but, due to the large theoretical error, there is still room for a large NP contribution, roughly of the order of [67]

$$
-4 \times 10^{-4} \lesssim\left(\frac{\epsilon^{\prime}}{\epsilon}\right)_{\mathrm{NP}} \lesssim+10 \times 10^{-4}
$$


We take this interval as a $1 \sigma$ range in our fits. The NP contribution coming from the VLQ can be computed from the simplified formula [42]

$$
\left(\frac{\epsilon^{\prime}}{\epsilon}\right)_{\mathrm{NP}}=P_{7} \operatorname{Im}\left(C_{7}^{s d}\right)
$$

where we consider only the dominant contribution coming from the operator $Q_{7}$. The Wilson coefficient at the electroweak scale

$$
C_{7}^{s d}=-\frac{\alpha}{6} \frac{\pi}{G_{F}^{2} m_{W}^{2} M_{B}^{2}} \frac{\tilde{Y}_{s}^{B} \tilde{Y}_{d}^{B^{*}}}{\lambda_{s d}^{u}},
$$

depends on the Yukawa coefficients defined in eq. (2.21). The coefficient in front is

$$
P_{7}=-102.02-1.32 B_{6}^{(1 / 2)}+2040.38 B_{8}^{(3 / 2)},
$$

where we use $B_{6}^{(1 / 2)}=1.36 \pm 0.23$ and $B_{8}^{(3 / 2)}=0.79 \pm 0.05$ [67] without considering the errors.

\section{Numerical results}

\subsection{Methods}

Here we describe the fit procedure. We use the same set of observables to perform the fit in three cases: (a) SM, (b) addition of one NB-VLQ and (c) addition of one generic VLQ. Therefore, these three cases can be compared directly. The fit is performed for $M_{B}=1.4 \mathrm{TeV}$, as allowed by direct search constraints (3.1). The observables considered in the fit are listed in table 2. For most of the observables, we assume gaussian likelihood and consider

$$
\chi^{2}=-2 \ln \mathcal{L}=\sum_{i} \frac{\left(\mathcal{O}_{i \text { theo }}-\mathcal{O}_{i \exp }\right)^{2}}{\sigma_{i}^{2}},
$$

where $\mathcal{O}_{i \text { theo }}$ is the functional form predicted by the model, $\mathcal{O}_{i \exp }$ is the central experimental value with error $\sigma_{i}$. The exception is the observable $\operatorname{Br}\left(K_{L} \rightarrow \mu^{+} \mu^{-}\right)$, listed in the end of table, which is treated differently from (4.1). We include it through the function

$$
f(x ; a, b, r)= \begin{cases}\left(\frac{a-x}{r(b-a)}\right)^{2}, & x \leq a \\ 0, & a<x<b \\ \left(\frac{x-b}{r(b-a)}\right)^{2}, & x \geq b\end{cases}
$$

which is flat in the range $x \in[a, b]$ but grows quadratically outside that range. The rate of growth is controlled by $r$, chosen to be $r=0.01$. For $x$ being $\operatorname{Br}\left(K_{L} \rightarrow \mu^{+} \mu^{-}\right)$, this function is added to the $\chi^{2}$ in (4.1) with the ranges $[a, b]$ listed in table 2 . The reason for the different treatment of this observable is that it is still subjected to large theoretical uncertainties but, nevertheless, strongly constrains certain BSM parameters. Therefore, we choose a function which does not contribute to the $\chi^{2}$ if the observable lies within the allowed range but quickly disfavors values which are outside that range. Regarding the absolute values of 


\begin{tabular}{|c|c|c|c|c|}
\hline Observable $\mathcal{O}_{i}$ & Experimental & SM (c.v [95\% CL]) & NB-VLQ & gen-VLQ \\
\hline$\left|V_{t b}\right|$ & $\begin{array}{c}1.013(30) \\
\text { eq. }(3.2)]\end{array}$ & $\begin{array}{c}0.99910 \\
{[0.99905,0.99916]}\end{array}$ & {$[0.9982,0.9992]$} & {$[0.9981,0.9991]$} \\
\hline $10^{10} \times B r\left(B_{d}^{0} \rightarrow \mu^{+} \mu^{-}\right),^{a}$ & $1.1_{-1.3}^{+1.4}[48]$ & $1.08[0.99,1.16]$ & {$[0.75,2.15]$} & {$[0.0,8.9]$} \\
\hline $10^{10} \times B r\left(K_{L} \rightarrow \mu^{+} \mu^{-}\right)_{\mathrm{SD}}$ & $<25[60]$ & $8.6[7.6,9.5]$ & {$[4.4,13.7]$} & {$[0,25]$} \\
\hline $10^{4} \times\left(\epsilon^{\prime} / \epsilon\right)_{\mathrm{NP}}$ & {$[-4,10][67]$} & - & {$[-6.0,6.6]$} & {$[-11,17]$} \\
\hline
\end{tabular}

${ }^{a}$ We use the central value for $f_{B_{d}}=192.0(4.3)$ [53] without the error.

Table 4. Observables predicted from the fit. The last two columns show the intervals at 95\% CL.

the CKM elements in (3.2), we do not consider the experimental values for $\left|V_{t d}\right|$ and $\left|V_{t s}\right|$, and instead we use the measured values for $\Delta m_{B_{q}}, q=d, s$, which receive contributions from the VLQ. We also symmetrize the errors and inflate the $1 \sigma$ ranges of (3.2) by $50 \%$ because the high precision of the measurements of the first row of the CKM matrix hints to unitarity violation if taken at face value [49-51]. The input parameters are listed in table 3. The parameters $f_{B_{d}} \hat{B}_{B_{d}}^{\frac{1}{2}}, f_{B_{s}} \hat{B}_{B_{s}}^{\frac{1}{2}}, f_{B_{s}}$ listed in this table, and $\eta_{c c}^{K}$ described in section 3.7, have large theoretical errors and thus are considered as nuisance parameters which are also included in the fit as in (4.1). We also consider the correlation between the first two of these nuisance parameters. To perform the minimization of the $\chi^{2}$ function and sampling the nearby region we use MultiNest [72-74] accessed through the PyTHON wrapper PyMultiNest [75]. The Wolfenstein parameters obtained from the SM fit are also listed in table 3. We can see they are similar to the fit from PDG in (3.5), although the values for $A, \bar{\rho}$ and $J$ are larger. The $95 \%$ CL interval for the observables considered in the fit are also shown in table 2 . We can see that the agreement with the experimental values are excellent. Moreover, our result for $B_{s}^{0} \rightarrow \mu^{+} \mu^{-}$is compatible with $\operatorname{Br}\left(B_{s}^{0} \rightarrow \mu^{+} \mu^{-}\right)=3.23(27) \times 10^{-9}$ of ref. [57] as well as with $\operatorname{Br}\left(B_{s}^{0} \rightarrow \mu^{+} \mu^{-}\right)=(3.41[3.01,3.81]) \times 10^{-9}$ from the fit in ref. [42]. Our intervals for $\Delta m_{B_{d}}$ and $\Delta m_{B_{s}}$ are also compatible with refs. [42, 79, 80]. The value of $\epsilon_{K}$ agrees with [81,82].

\subsection{Results}

In table 4 we show observables that are predicted from the fit. We extract only the interesting observables and compare the case of SM, one NB-VLQ and one generic VLQ. For all these cases we show the $95 \% \mathrm{CL}$ interval for $M_{B}=1.4 \mathrm{TeV}$. We can see that the value for $\left|V_{t b}\right|$ can indeed be lowered with the presence of one VLQ [52] but probing it will require a precision which is hard to attain. For the rare decay $B_{d}^{0} \rightarrow \mu^{+} \mu^{-}$, our value obtained for the $\mathrm{SM}$ is very close to the one obtained in ref. [57], $\operatorname{Br}\left(B_{d}^{0} \rightarrow \mu^{+} \mu^{-}\right)=1.06(9) \times 10^{-10}$. In the presence of one generic VLQ, a large interval is still allowed. For the NB case, the allowed interval is larger than the SM but narrower than the generic case. For the kaon decay $K_{L} \rightarrow \mu^{+} \mu^{-}$, all the values up to the upper limit can be reached for the generic VLQ case but for the NB-VLQ the interval shrinks to a narrower interval. Both these intervals are expectedly wider than for the SM. A similar conclusion also applies to the VLQ contribution to $\epsilon^{\prime} / \epsilon$ : a large contribution is still possible for the generic case 
but a much smaller contribution is possible for the NB case. In fact, in the NB case, the predicted maximal contribution at 95\% CL is smaller than allowed experimentally. The obtained intervals differ from ref. [42] because of the updated calculation reported in ref. [67]. We show in figures 1,2 and 3 the allowed regions for the combination $\tilde{Y}_{i}^{B} \tilde{Y}_{j}^{B *}$, cf. $(2.21)$, in the complex plane for the sectors $(i j)=(s d),(b d),(b s)$, respectively. These quantities were denoted as $\Lambda_{i j}$ in ref. [42] and can be compared here. The region in yellow (orange) corresponds to the generic VLQ case at 95\% CL (68\% CL) while the region in blue corresponds to the NB-VLQ case at $95 \%$ CL. In all sectors the allowed regions for the NB-VLQ case are much smaller than the generic case due to the correlations of the model. In the sectors $(s d)$ and $(b d)$, the $95 \%$ CL region for the NB case is even smaller than the $65 \%$ CL region of the generic case. For comparison, we also show in a dashed contour the approximate $95 \%$ CL regions from the global fit in ref. [42] which used $M_{B}=1 \mathrm{TeV}$ (our value is $\left.M_{B}=1.4 \mathrm{TeV}\right)$. Some comments are in order.

- For the $(s d)$ sector, we use the most constraining observables as in ref. [42]: $\operatorname{Br}\left(K_{L} \rightarrow\right.$ $\mu \bar{\mu})_{\mathrm{SD}}$ and $\left(\epsilon^{\prime} / \epsilon\right)_{\mathrm{NP}}$. The region is constrained horizontally and vertically, respectively, by these observables. Our implementation of $\operatorname{Br}\left(K_{L} \rightarrow \mu \bar{\mu}\right)_{\mathrm{SD}}$, however, is different and we obtain a region wider horizontally. Vertically, we consider an updated (2020) constraint for $\left(\epsilon^{\prime} / \epsilon\right)_{\mathrm{NP}}$ [67] which differs from the constraint in ref. [42] of 2016. The net result is an upward shift in the region. The region is possibly wider due to the change in the VLQ mass from $1 \mathrm{TeV}$ to $1.4 \mathrm{TeV}$.

- For the $(b d)$ sector, our region for the generic case is much larger than the region found in ref. [42] because we do not include the observable $\operatorname{Br}\left(B^{+} \rightarrow \pi^{+} \mu \bar{\mu}\right)$ which is the most constraining for that mass range. Nevertheless, the regions are roughly compatible and our region for NB case is entirely contained.

- For the (bs) sector, we use the most constraining observables as in ref. [42]: $\operatorname{Br}\left(B_{s} \rightarrow\right.$ $\mu \bar{\mu})$. The obtained region for the generic case is largely compatible, with possible enlargement due to the increase in $M_{B}$. For the NB case, the region is much narrower in the imaginary part.

Note that, after we factor $V_{d_{L}}$, the mixing $\Theta_{i}$ in (2.12) of up-quarks with the heavy $B$ quark through $W$ exchange is given by

$$
\Theta_{i}=\frac{v}{\sqrt{2}} \tilde{Y}_{i}^{B} / M_{B}
$$

i.e., it is proportional to $\tilde{Y}_{i}^{B}$ in (2.21); see eqs. (2.13) and (2.14). So the constraints in figures 1, 2 and 3 can be interpreted as constraints on $\Theta_{i} \Theta_{j}^{*}$. Similarly, flavor changing neutral currents couple to the $Z$ boson through $X^{d}$ in (2.15), which is proportional to

$$
X_{i j}^{d} \approx-\Theta_{i} \Theta_{j}^{*}=-\frac{v^{2}}{2 M_{B}^{2}}\left(\tilde{Y}_{i}^{B} \tilde{Y}_{j}^{B *}\right), \quad i \neq j .
$$

The approximation refers to the leading seesaw approximation. The factor inside the parentheses is exactly the quantity appearing in figures 1,2 and 3. Indeed, if we link the 


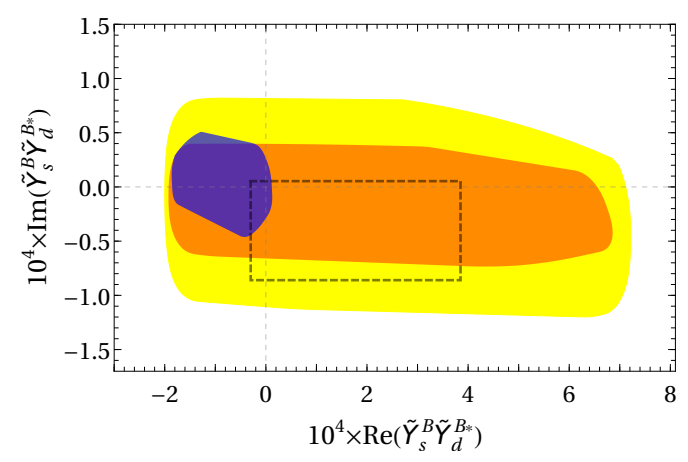

Figure 1. Allowed regions for $\tilde{Y}_{s}^{B} \tilde{Y}_{d}^{B *}$ for one generic VLQ (95\% CL in yellow, $68 \%$ CL in orange) and one NB-VLQ (95\% CL in blue) for $M_{B}=1.4 \mathrm{TeV}$. The approximate $95 \%$ CL region extracted from the global fit in ref. [42] for $M_{B}=1 \mathrm{TeV}$ is also shown (dashed contour).

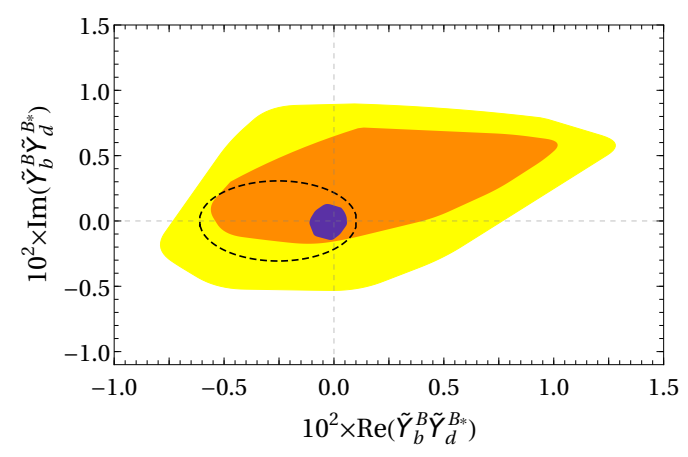

Figure 2. Allowed regions for $\tilde{Y}_{b}^{B} \tilde{Y}_{d}^{B *}$ for one generic VLQ (95\% CL in yellow, $68 \%$ CL in orange) and one NB-VLQ (95\% CL in blue) for $M_{B}=1.4 \mathrm{TeV}$. The approximate 95\% CL region extracted from the global fit in ref. [42] for $M_{B}=1 \mathrm{TeV}$ is also shown (dashed contour).

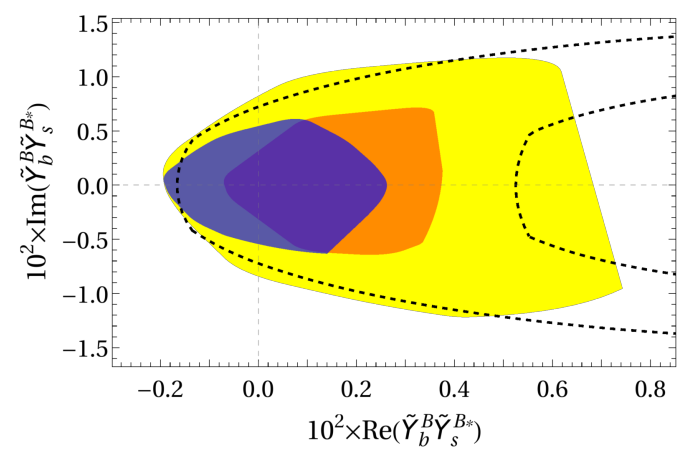

Figure 3. Allowed regions for $\tilde{Y}_{b}^{B} \tilde{Y}_{s}^{B *}$ for one generic VLQ (95\% CL in yellow, $68 \%$ CL in orange) and one NB-VLQ (95\% CL in blue) for $M_{B}=1.4 \mathrm{TeV}$. The approximate 95\% CL region extracted from the global fit in ref. [42] for $M_{B}=1 \mathrm{TeV}$ is also shown (dashed contour). 
flavor changing physics scale in (1.1) to $1 / \Lambda_{i j}^{2} \sim\left|\tilde{Y}_{i}^{B} \tilde{Y}_{j}^{B *}\right| / M_{B}^{2}$, apart from a global factor, we can extract from the figures the milder hierarchies for the NB case,

$$
\Lambda_{s d}^{-1}: \Lambda_{b d}^{-1}: \Lambda_{b s}^{-1} \sim 0.18: 0.4: 1 .
$$

In part, this is due to a larger set of observables we consider, which is not restricted to $\Delta F=2$ observables. It is important to emphasize that the NB scheme leads to a hierarchy of $\tilde{Y}_{i}^{B}$ inherited from the SM Yukawa couplings but the overall scale is not determined [15]. In special, such a scale is not suppressed by the bottom Yukawa, such as in the MFV setting, and is only limited by perturbativity from the theoretical side while flavor observables in the (bs) sector, cf. figure 3, allows $\left|\tilde{Y}_{b}^{B}\right| \sim 0.3$ for $M_{B}=1.4 \mathrm{TeV}$, considering that $\left|\tilde{Y}_{b}^{B} \tilde{Y}_{s}^{B *}\right| \sim\left|V_{t s}\left(\tilde{Y}_{b}^{B}\right)^{2}\right|$. For larger $M_{B}$, larger values are allowed. See appendix B for approximate formulas for $\tilde{Y}^{B}$.

There is one frequently considered simplified scenario where the combinations appearing in figures 1, 2 and 3 all vanish and no constraint can be extracted. That is the case where the heavy quark $B$ only couples with the third SM family [55] and

$$
\tilde{Y}^{B}=\left(0,0, y_{B}\right)^{\top} .
$$

Similar conclusions apply to scenarios where $B$ couples only to the first family or only to the second family. For these cases, it is more interesting to show directly the constraint on $\left|V_{i B}\right| \approx\left|\left(V_{d_{L}} \Theta\right)_{i}\right|$, which also quantifies the deviation from unitarity for the $3 \times 3$ sub-block. We show the allowed regions in figure 4 . The color code is the same as in figure 1. We can see that for the generic case (yellow/orange) all three $\left|V_{i B}\right|$ can reach values of the same order of magnitude (not simultaneously) but for the NB case (blue), the allowed values are hierarchical for $i=t, c, u$. The latter pattern is compatible with the hierarchy (2.17) but it follows (2.18) only roughly. Therefore, it is clear that $\left|V_{t B}\right|$ (similarly $\left|Y_{3}^{B}\right|$ ) can reach values as large as in the generic case but the other mixings (Yukawas) are automatically suppressed. The constraint for the mixing $\left|V_{i B}\right|$ has another advantage: it is more applicable to different VLQ masses. The reason is that our flavor observables are essentially sensitive to $X^{d}$ which depends on the CKM matrix $V$. The mixing $V_{i B}$, however, scales approximately as (2.14) and a larger mass implies a smaller mixing for the same Yukawa $Y^{B}$. So the constraints in figures 1,2 and 3 can be approximately adapted to other VLQ masses after rescaling while the constraints on figure 4 can be approximately applied to any mass. ${ }^{12}$ Concerning CP violation, we show in figure 5 the $95 \%$ CL intervals for some Jarlskog invariants involving only the $3 \times 3$ sub-block of CKM. The definition we use is $[15]$

$$
J_{i j k l}=\operatorname{Im}\left[V_{i j} V_{j k}^{\dagger} V_{k l} V_{l i}^{\dagger}\right] .
$$

The usual Jarlskog invariant for the SM is $J_{u s c b}=J_{1223}$ and we show in the gray band the allowed 95\% interval obtained from the fit. With the presence of one VLQ, the CKM matrix becomes $3 \times 4$ and the Jarlskog invariants are not all the same. We also show the intervals

\footnotetext{
${ }^{12}$ This scaling breaks down for masses larger than tens of $\mathrm{TeV}$ due to the one-loop box contribution that starts to dominate the mixings of neutral mesons [83].
} 

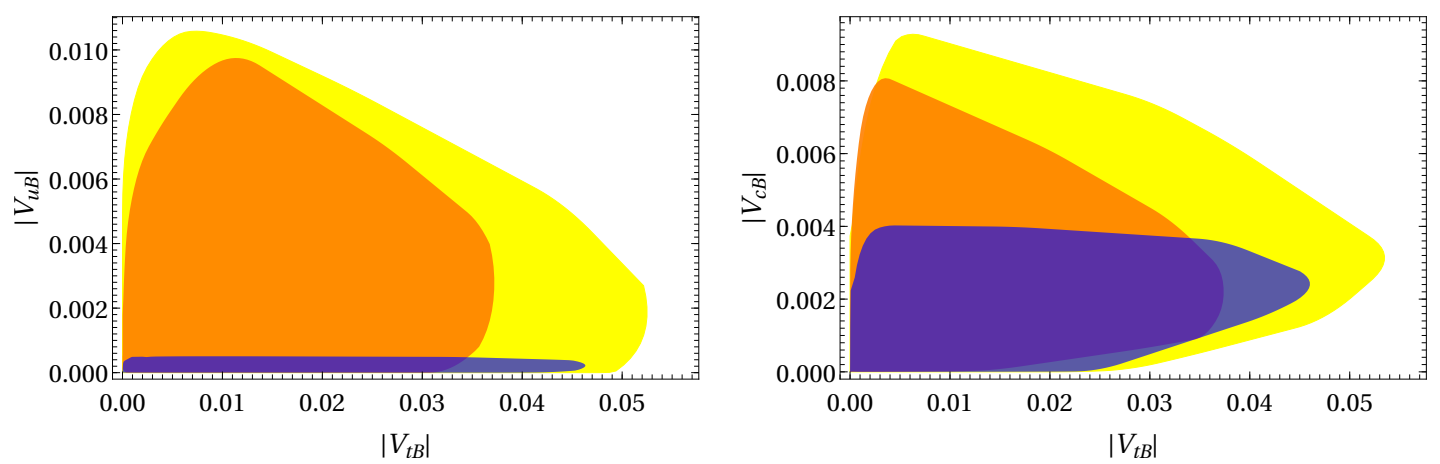

Figure 4. Allowed regions of $\left|V_{i B}\right|$ for one generic VLQ (95\% in yellow, $68 \%$ in orange) and one NB-VLQ (95\% CL in blue).

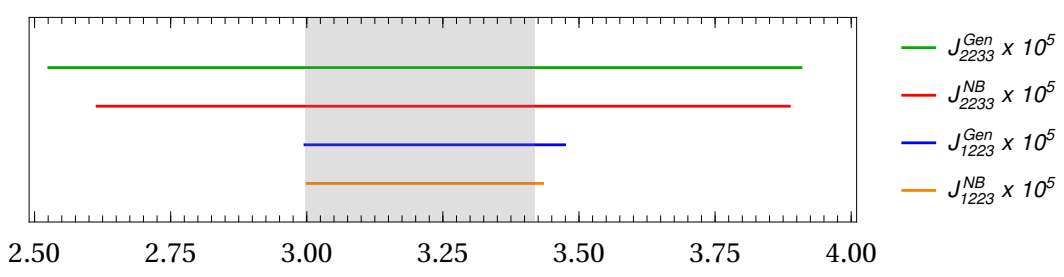

Figure 5. 95\% CL intervals for Jarlskog invariants, cf. (4.7), for the case of one generic VLQ and one NB-VLQ. The gray band is the $95 \%$ CL interval for the SM.

for $J_{1223}$ and $J_{2233}$ for a generic VLQ and for a NB-VLQ. The invariant $J_{2233}$ was shown in ref. [15] to present a larger variation for one NB-VLQ. We can indeed see in figure 5 that the interval for $J_{2233}$ with one VLQ is larger than in the SM for both the generic and the NB case. The deviation of $J_{2233}$ from the SM value we see in figure 5 could be tested in the future by a more precise determination of $\phi_{s}$ proportional to the angle between $V_{c s} V_{c b}^{*}$ and $V_{t s} V_{t b^{*}}$ which enters precisely in $J_{2233}=\operatorname{Im}\left[V_{c s} V_{t s}^{*} V_{t b} V_{c b}^{*}\right]$. Currently, the errors are no smaller than $40 \%$ [84-86] but the precision at LHCb at High-luminosity LHC with $300 \mathrm{fb}^{-1}$ is expected to be around $10 \%$. [87]. Concerning the 5 BSM parameters (2.10) of our seesaw parametrization for one NB-VLQ, there are essentially no constraints on the parameters $b, \beta_{1}, \beta_{2}, \gamma$. The mass $M_{B}$ can be constrained in direct searches in colliders but are not directly constrained by flavor observables which are dependent on the mixing between the SM quarks and the VLQ. For a qualitative comparison, we briefly discuss the case of one up-type NB-VLQ in appendix D. The salient features are the same: the Yukawa couplings $Y_{i}^{T}$ of the heavy up-type VLQ $T_{L, R}$ with the SM quarks are hierarchical reflecting the hierarchy in the up-type Yukawas and in the CKM mixing. For this reason, for $M_{T} \sim 1.3 \mathrm{TeV}$, obeying flavor diagonal electroweak precision constraints automatically suppresses flavor changing observables involving lighter families. 


\section{Conclusions}

By performing a global fit on interesting low energy flavor observables we constrain the possibilities for the presence of one VLQ of charge $-1 / 3$. We compare the case of one generic VLQ and one VLQ of Nelson-Barr type. Only the latter would be responsible for transmitting the spontaneous $\mathrm{CP}$ violation from a $\mathrm{CP}$ breaking sector to the $\mathrm{SM}$ in the Nelson-Barr setting. We see that, using flavor observables, distinguishing the case of one NB-VLQ from a generic VLQ of down type will be a challenging task. Nevertheless the presence of one NB-VLQ is definitely falsifiable once a VLQ is found by other means and its flavor signature lies in a region of parameter space outside of what is allowed for one NBVLQ but within the region allowed for one generic VLQ. The results presented in table 4 and figures 1, 2 and 3 show that the parameter space constrained by flavor observables is significantly smaller for the NB case compared to the generic case due to the inherent correlations that appear in the model, a feature which was uncovered in ref. [15]. In the process, we update the allowed parameter space for the presence of one generic VLQ, which can be directly compared to the regions obtained in ref. [42]. These constraints should apply to all limiting cases where one VLQ of down type is present. For example, the often considered case of a VLQ coupling only to the third family is just a subcase and must have the allowed parameter space contained in the generic region. On the other hand, the NB case is a specific example of a scenario where the VLQ couples dominantly with the third family but also couples to the lighter families with hierarchically smaller couplings. In summary, scenarios involving VLQs of Nelson-Barr type are simple scenarios where the presence of VLQs are motivated to solve the strong CP problem. Distinguishing a VLQ of NB type from a VLQ unrelated to the origins of CP breaking in nature is a very difficult but interesting task that warrants further investigation.

\section{Acknowledgments}

C.C.N. acknowledges partial support by Brazilian Fapesp, grant 2014/19164-6, and CNPq, grant 304262/2019-6. G.D.C. acknowledges financial support by the Coordenação de Aperfeiçoamento de Pessoal de Nível Superior - Brasil (CAPES) — Finance Code 001. We thank Andre Lessa for helpful comments.

\section{A Inversion formula}

Here we briefly review the inversion formula for (2.5c) developed in ref. [15] for the seesaw parametrization of one NB-VLQ. The Yukawa couplings $Y^{d}$ in (2.5c) and $Y^{B}$ in (2.5b) depend on the quantities $\mathscr{Y}^{d}$ and $w$ but $Y^{d}$ should be in accord with SM input (2.6). The inversion formula allows us to take the latter into account automatically, leaving $\mathscr{Y}^{d}$ and $w$ dependent on $\left\{b, \gamma, \beta_{1}, \beta_{2}\right\}$. The VLQ mass $M_{B}$ completes the five free BSM parameters. Given $\beta_{1}, \beta_{2}$ in $(2.9), \mathscr{Y}^{d}$ in $(2.5 \mathrm{c})$ can be determined as

$$
\mathscr{Y}^{d}=A \mathcal{O} B^{-1}
$$


where

$$
\begin{aligned}
& A=\left(\operatorname{Re}\left(Y^{d} Y^{d^{\dagger}}\right)\right)^{1 / 2}, \\
& B=\operatorname{diag}\left(1, \sqrt{1-b^{2}}, \sqrt{1-a^{2}}\right),
\end{aligned}
$$

and the orthogonal matrix $\mathcal{O}$ will be defined below. The matrix $B$ contains one free parameter, chosen to be $b$, while $a$ is fixed from

$$
\frac{a}{\sqrt{1-a^{2}}} \frac{b}{\sqrt{1-b^{2}}}=\mu \text {. }
$$

The value of $0<\mu<1$ comes from the real antisymmetric matrix

$$
C \equiv A^{-1} \operatorname{Im}\left(Y^{d} Y^{d^{\dagger}}\right) A^{-1}
$$

with eigenvalues $(0, i \mu,-i \mu)$. The real orthogonal matrix $\mathcal{O}$ in the formula (A.1) transforms $C$ to the canonical form

$$
\mathcal{O}^{\top} C \mathcal{O}=\mu\left(\begin{array}{ccc}
1 & & \\
& 0 & -1 \\
1 & 0
\end{array}\right) .
$$

This matrix $\mathcal{O}$ is not unique as (A.5) is invariant by additional rotation on the $2-3$ plane. Then, we can write

$$
\mathcal{O}=\mathcal{O}_{0}\left(\begin{array}{ccc}
1 & & \\
& \cos \gamma & \sin \gamma \\
& -\sin \gamma & \cos \gamma
\end{array}\right),
$$

where $\mathcal{O}_{0}=\left(e_{1}\left|e_{2}\right| e_{3}\right)$ is a representative matrix satisfying (A.5). The first column vector $e_{1}$ is fixed as the eigenvector of $C$ with eigenvalue zero. The sign is determined so that $\mathscr{Y}_{11}^{d}>0$. For our numerical code, we choose $e_{2}$ and $e_{3}$ so that $u_{-}=e_{2}+i e_{3}$ is the eigenvector of $C$ with eigenvalue $-i \mu$. We choose the convention that $u_{-}$has real positive first component so that $e_{3}$ has vanishing first component. As for the parameter ranges, the angular and phase variables, $\gamma, \beta_{1}, \beta_{2}$, should all be confined to $[0,2 \pi]$. The $b$ parameter is confined to the range (2.11) so that the quality of the seesaw approximation is guaranteed within $1 \%$.

\section{B Approximate formulas for the Yukawas}

The inversion formula reviewed in appendix A solves for $\mathscr{Y}^{d}$ in $(2.5 \mathrm{c})$ in terms of the SM Yukawa $Y^{d}$ and this relation is exact in the leading seesaw approximation. We can obtain approximate relations for $Y^{B}$ which makes explicit the hierarchy of these couplings. Substituting the solution (A.1) in (2.5b) we obtain

$$
Y^{B}=\left[\operatorname{Re}\left(Y^{d} Y^{d^{\dagger}}\right)\right]^{1 / 2} \mathcal{O}\left(\begin{array}{c}
0 \\
i x_{b} \\
\frac{\mu}{x_{b}}
\end{array}\right),
$$


where $x_{b} \equiv \frac{b}{\sqrt{1-b^{2}}}$ and we have used (A.3). Considering $Y^{d}$ is hierarchical and ignoring the real part, we obtain for $b \leq 1 / \sqrt{2}$ the approximate bound

$$
\left|Y_{i}^{B}\right| \lesssim y_{b}\left|V_{i b}\right| \frac{\mu}{x_{b}} \sim \frac{\mu}{x_{b}} \times\left(10^{-4}, 10^{-3}, 0.024\right) .
$$

Since $\left|V_{i B}\right| \approx\left|\left(\theta_{L}\right)_{i}\right|=\frac{v}{\sqrt{2}}\left|Y_{i}^{B}\right| / M_{B}$, this expression describes to a good approximation the upper boundary of figure 3 in ref. [15] as a function of $b$ (typically $\mu \sim 1$ ). As the CKM matrix is hierarchical, we can also write $\left|\tilde{Y}_{i}^{B}\right| \sim\left|Y_{i}^{B}\right|$. Equation (B.2) clearly shows the hierarchy among the couplings $Y_{i}^{B}$. It also shows that the absolute scale of the Yukawa couplings of the NB-VLQ are not necessarily suppressed by the bottom Yukawa for $x_{b} \ll 1$. Indeed, our fit showed that it can reach $\left|Y_{3}^{B}\right| \sim y_{b} / x_{b} \sim 0.3$ for $M_{B}=1.4 \mathrm{TeV}$ in conformity with other fits of the generic VLQ case [42]. For completeness, we can quantify the quality of the leading seesaw approximation by considering the deviation of the real heaviest mass eigenvalue of (2.3) compared to the input mass $M_{B}$ :

$$
\frac{\delta M_{B}}{M_{B}} \approx \frac{1}{2}\left|\theta_{L}\right|^{2} \lesssim \frac{m_{b}^{2}}{2 M_{B}^{2}} \frac{\mu^{2}}{x_{b}^{2}}
$$

The mixing $\theta_{L}$ is related to $Y^{B}$ by (2.14). With this relation, one can limit the maximum deviation allowed by setting a lower limit for $b$.

\section{Auxiliary functions}

The Inami-Lim functions [88] are

$$
\begin{aligned}
S_{0}(x, y) & =\frac{x\left(x^{2}-8 x+4\right) y \log (x)}{4(x-1)^{2}(x-y)}+\frac{x\left(y^{2}-8 y+4\right) y \log (y)}{4(y-1)^{2}(y-x)}-\frac{3 x y}{4(x-1)(y-1)} \\
S_{0}(x) & =\frac{x^{3}-11 x^{2}+4 x}{4(1-x)^{2}}-\frac{3 x^{3} \log (x)}{2(1-x)^{3}} \\
Y_{0}(x) & =\frac{3 x^{2} \log (x)}{8(x-1)^{2}}-\frac{3 x}{8(x-1)}+\frac{x}{8} .
\end{aligned}
$$

We follow the convention of ref. [52]. Note that $S_{0}(x)=\lim _{y \rightarrow x} S_{0}(x, y)$.

\section{One up-type NB-VLQ}

In this case, the down-type VLQs $B_{L, R}$ are replaced by up-type VLQs $T_{L, R}$ and the Lagrangians (2.1) (real basis) and (2.2) (generic basis) can be adapted accordingly. The Yukawa couplings that will be constrained by flavor observables are

$$
-\mathscr{L} \subset \tilde{Y}_{i}^{T} \bar{q}_{i L} \tilde{H} T_{R}
$$

in the basis where $Y^{u}$ is diagonal and $Y^{d}$ carries the CKM of the SM. Note that $T$ in $\tilde{Y}^{T}$ is a label and not the transpose operation. To avoid confusion, we will omit this label in the following discussion. Similarly, $Y=Y^{T}$ without the tilde is the same Yukawa in the 
basis where $Y^{d}$ is diagonal. Because of CKM hierarchy, $\left|\tilde{Y}_{i}\right| \sim\left|Y_{i}\right|$. The full CKM matrix will be $4 \times 3$ of the form

$$
V \approx\left(\frac{\mathbb{1}_{3}-\frac{1}{2} \Theta \Theta^{\dagger}}{\Theta^{\dagger}}\right) V_{u_{L}}^{\dagger},
$$

where $V_{u_{L}}^{\dagger}=V_{c k m}$ is the $3 \times 3 \mathrm{CKM}$ matrix within the SM, except for possible two additional phases. The coupling $\tilde{Y}$ controls the mixing of the VLQ with the SM quarks as, in leading order,

$$
\Theta_{i}=\frac{v}{\sqrt{2}} \tilde{Y}_{i} M_{T}^{-1}
$$

The FCNC coupling to the $Z$ now depends on $X^{u}=V V^{\dagger}$. In order to reproduce the SM up-type Yukawa couplings, a relation analogous to (2.5c) needs to be satisfied and the up-type NB-VLQ will also couple to SM quarks hierarchically. ${ }^{13}$ Owing to this hierarchy in the VLQ mixing, we can use the constraints from the case where the VLQ couples only to the third family. For example, the oblique parameters $S, T$ constrain [55]

$$
\left|V_{T b}\right| \approx\left|\Theta_{3}\right| \lesssim 0.12
$$

for $M_{T}>1.3 \mathrm{TeV}$. This leads to

$$
\left|\tilde{Y}_{3}\right| \lesssim 0.9 \times\left(\frac{M_{T}}{1.3 \mathrm{TeV}}\right)
$$

This constraint is stronger than other electroweak observables that deviate due to modified $Z b b$ coupling. Adapting the approximate relation (B.2) to an up-type NB-VLQ, we obtain

$$
\left|Y_{i}\right| \lesssim y_{t}\left|V_{t i}^{*}\right| \frac{\mu}{x_{b}} \sim \frac{\mu}{x_{b}} \times(0.009,0.04,1)
$$

Therefore, if we keep the hierarchy in (D.6), ${ }^{14}$ the constraint (D.5) naturally translates into a much stronger constraint on the other Yukawas:

$$
\left|\tilde{Y}_{1}\right| \lesssim 0.008 \times\left(\frac{M_{T}}{1.3 \mathrm{TeV}}\right), \quad\left|\tilde{Y}_{2}\right| \lesssim 0.04 \times\left(\frac{M_{T}}{1.3 \mathrm{TeV}}\right)
$$

So due to the hierarchy of $\tilde{Y}$, analogously to the down-type NB-VLQ, the Yukawa couplings of the up-type NB-VLQ with lighter quarks are automatically suppressed. As a result, flavor changing observables involving lighter quarks may not be as relevant as for a generic up-type VLQ without the hierarchy in the couplings. Ref. [83] analyzed various flavor observables for all VLQ representations coupling to the SM. Due to the hierarchy in (D.6), the constraint (D.5) coming from flavor diagonal electroweak precision observables is more

\footnotetext{
${ }^{13}$ Note that due to $y_{t} \gg y_{b}$, the leading seesaw approximation is much less precise for an up-type NBVLQ than for a down-type NB-VLQ, cf. (B.3). But once we consider (D.4), the approximation is better than $1 \%$.

${ }^{14}$ Strictly speaking, this hierarchy may be subjected to an order of magnitude variation as the Yukawas do not need to saturate the upper limits in (D.6); see figure 3 in ref. [15] for the down-type NB-VLQ. In fact, to respect (D.5), $Y_{3}$ will be far from saturating the upper limit.
} 
important than flavor violating ones. For example, the decay $D^{0} \rightarrow \mu^{+} \mu^{-}$constrains the combination [83]

$$
\left|\tilde{Y}_{1} \tilde{Y}_{2}^{*}\right|<0.11 \times\left(\frac{M_{T}}{1.3 \mathrm{TeV}}\right)^{2}
$$

But the combination of the limits in (D.7) is much stronger. Analogous considerations apply to other limits. Although not very important for $M_{T} \sim \mathrm{TeV}$, the one-loop contributions to neutral meson mixings may become important for larger masses because the amplitudes scale as $Y^{4} / M_{T}^{2}$ (box diagram) instead of $Y^{2} / M_{T}^{2}$. Let us collect the results of ref. [83]:

$$
\begin{aligned}
\left|\operatorname{Im} Y_{1} Y_{2}^{*}\right| & <0.002 \times \frac{M_{T}}{1.3 \mathrm{TeV}}, \\
\left|\operatorname{Re} Y_{1} Y_{2}^{*}\right| & <0.03 \times \frac{M_{T}}{1.3 \mathrm{TeV}}, \\
\left|Y_{1} Y_{3}^{*}\right| & <0.05 \times \frac{M_{T}}{1.3 \mathrm{TeV}}, \\
\left|Y_{2} Y_{3}^{*}\right| & <0.2 \times \frac{M_{T}}{1.3 \mathrm{TeV}} .
\end{aligned}
$$

Notice the different scaling in $M_{T}$. The last two constraints become more important than (D.5), combined with (D.6), for $M_{T} \sim 7 \mathrm{TeV}$.

Open Access. This article is distributed under the terms of the Creative Commons Attribution License (CC-BY 4.0), which permits any use, distribution and reproduction in any medium, provided the original author(s) and source are credited.

\section{References}

[1] A.E. Nelson, Naturally Weak CP-violation, Phys. Lett. B 136 (1984) 387 [InSPIRE].

[2] S.M. Barr, Solving the Strong CP Problem Without the Peccei-Quinn Symmetry, Phys. Rev. Lett. 53 (1984) 329 [INSPIRE].

[3] H. Georgi, A Model of Soft CP-violation, Hadronic J. 1 (1978) 155 [InSPIRE].

[4] S.M. Barr and P. Langacker, A Superweak Gauge Theory of CP Violation, Phys. Rev. Lett. 42 (1979) 1654 [INSPIRE].

[5] M.A.B. Beg and H.S. Tsao, Strong P, T Noninvariances in a Superweak Theory, Phys. Rev. Lett. 41 (1978) 278 [INSPIRE].

[6] R.N. Mohapatra and G. Senjanović, Natural Suppression of Strong p and t Noninvariance, Phys. Lett. B 79 (1978) 283 [INSPIRE].

[7] G. Segre and H.A. Weldon, Natural Suppression of Strong $P$ and T Violations and Calculable Mixing Angles in $\mathrm{SU}(2) \otimes \mathrm{U}(1)$, Phys. Rev. Lett. 42 (1979) 1191 [InSPIRE].

[8] M. Dine and P. Draper, Challenges for the Nelson-Barr Mechanism, JHEP 08 (2015) 132 [arXiv:1506.05433] [INSPIRE].

[9] G. Perez and A. Shalit, High quality Nelson-Barr solution to the strong CP problem with $\theta=\pi$, JHEP 02 (2021) 118 [arXiv:2010.02891] [INSPIRE]. 
[10] M. Pospelov and A. Ritz, Electric dipole moments as probes of new physics, Annals Phys. 318 (2005) 119 [hep-ph/0504231] [INSPIRE].

[11] J.M. Pendlebury et al., Revised experimental upper limit on the electric dipole moment of the neutron, Phys. Rev. D 92 (2015) 092003 [arXiv: 1509.04411] [INSPIRE].

[12] C.A. Baker et al., An Improved experimental limit on the electric dipole moment of the neutron, Phys. Rev. Lett. 97 (2006) 131801 [hep-ex/0602020] [INSPIRE].

[13] B. Graner, Y. Chen, E.G. Lindahl and B.R. Heckel, Reduced Limit on the Permanent Electric Dipole Moment of Hg199, Phys. Rev. Lett. 116 (2016) 161601 [Erratum ibid. 119 (2017) 119901] [arXiv:1601.04339] [INSPIRE].

[14] G. Hiller and M. Schmaltz, Solving the Strong CP Problem with Supersymmetry, Phys. Lett. $B \mathbf{5 1 4}$ (2001) 263 [hep-ph/0105254] [INSPIRE].

[15] A.L. Cherchiglia and C.C. Nishi, Consequences of vector-like quarks of Nelson-Barr type, JHEP 08 (2020) 104 [arXiv:2004.11318] [INSPIRE].

[16] L. Lavoura, Solution of the strong CP problem, Phys. Lett. B 391 (1997) 441 [hep-ph/9611266] [INSPIRE].

[17] L. Bento, G.C. Branco and P.A. Parada, A Minimal model with natural suppression of strong CP-violation, Phys. Lett. B 267 (1991) 95 [inSPIRE].

[18] A.L. Cherchiglia and C.C. Nishi, Solving the strong CP problem with non-conventional CP, JHEP 03 (2019) 040 [arXiv: 1901.02024] [INSPIRE].

[19] D. Egana-Ugrinovic, S. Homiller and P. Meade, Aligned and Spontaneous Flavor Violation, Phys. Rev. Lett. 123 (2019) 031802 [arXiv:1811.00017] [INSPIRE].

[20] C. Cheung, A.L. Fitzpatrick and L. Randall, Sequestering CP-violation and GIM-Violation with Warped Extra Dimensions, JHEP 01 (2008) 069 [arXiv:0711.4421] [INSPIRE].

[21] S.M. Barr, Supersymmetric solutions to the strong CP problem, Phys. Rev. D 56 (1997) 1475 [hep-ph/9612396] [INSPIRE].

[22] L. Vecchi, Spontaneous CP-violation and the strong CP problem, JHEP 04 (2017) 149 [arXiv: 1412.3805] [INSPIRE].

[23] J. Schwichtenberg, P. Tremper and R. Ziegler, A grand-unified Nelson-Barr model, Eur. Phys. J. C 78 (2018) 910 [arXiv:1802.08109] [InSPIRE].

[24] Y. Mimura, R.N. Mohapatra and M. Severson, Grand unified parity solution to the strong CP problem, Phys. Rev. D 99 (2019) 115025 [arXiv: 1903.07506] [InSPIRE].

[25] G. Choi and T.T. Yanagida, Solving the strong CP problem with horizontal gauge symmetry, Phys. Rev. D 100 (2019) 095023 [arXiv: 1909.04317] [INSPIRE].

[26] J. Evans, C. Han, T.T. Yanagida and N. Yokozaki, Complete solution to the strong CP problem: Supersymmetric extension of the Nelson-Barr model, Phys. Rev. D 103 (2021) L111701 [arXiv: 2002.04204] [INSPIRE].

[27] S.L. Glashow, A Simple solution to the strong CP problem, hep-ph/0110178 [INSPIRE].

[28] S. Antusch, M. Holthausen, M.A. Schmidt and M. Spinrath, Solving the Strong CP Problem with Discrete Symmetries and the Right Unitarity Triangle, Nucl. Phys. B 877 (2013) 752 [arXiv: 1307.0710] [INSPIRE]. 
[29] D. Chang and W.-Y. Keung, A New class of solutions to the strong CP problem with a small two loop theta, Phys. Rev. D 70 (2004) 051901 [hep-ph/0312139] [INSPIRE].

[30] F. Björkeroth, F.J. de Anda, I. de Medeiros Varzielas and S.F. King, Towards a complete $A_{4} \times \mathrm{SU}(5)$ SUSY GUT, JHEP 06 (2015) 141 [arXiv:1503.03306] [INSPIRE].

[31] M. Perelstein, M.E. Peskin and A. Pierce, Top quarks and electroweak symmetry breaking in little Higgs models, Phys. Rev. D 69 (2004) 075002 [hep-ph/0310039] [INSPIRE].

[32] M. Schmaltz and D. Tucker-Smith, Little Higgs review, Ann. Rev. Nucl. Part. Sci. 55 (2005) 229 [hep-ph/0502182] [INSPIRE].

[33] R. Contino, L. Da Rold and A. Pomarol, Light custodians in natural composite Higgs models, Phys. Rev. D 75 (2007) 055014 [hep-ph/0612048] [INSPIRE].

[34] R. Contino, T. Kramer, M. Son and R. Sundrum, Warped/composite phenomenology simplified, JHEP 05 (2007) 074 [hep-ph/0612180] [INSPIRE].

[35] O. Matsedonskyi, G. Panico and A. Wulzer, Light Top Partners for a Light Composite Higgs, JHEP 01 (2013) 164 [arXiv:1204.6333] [INSPIRE].

[36] D.B. Kaplan, Flavor at SSC energies: A New mechanism for dynamically generated fermion masses, Nucl. Phys. B 365 (1991) 259 [InSPIRE].

[37] G. Isidori, Flavor physics and CP-violation, in proceedings of the 2012 European School of High-Energy Physics, La Pommeraye, Anjou, France, 6-19 June 2012, pp. 69-105 [arXiv: 1302.0661] [INSPIRE].

[38] G. Isidori, Y. Nir and G. Perez, Flavor Physics Constraints for Physics Beyond the Standard Model, Ann. Rev. Nucl. Part. Sci. 60 (2010) 355 [arXiv:1002.0900] [InSPIRE].

[39] G.C. Branco and L. Lavoura, On the Addition of Vector Like Quarks to the Standard Model, Nucl. Phys. B 278 (1986) 738 [InSPIRE].

[40] G.C. Branco, L. Lavoura and J.P. Silva, CP Violation, Oxford University Press, Oxford U.K. (1999).

[41] Y. Nir, CP violation in and beyond the standard model, hep-ph/9911321 [INSPIRE].

[42] C. Bobeth, A.J. Buras, A. Celis and M. Jung, Patterns of Flavour Violation in Models with Vector-Like Quarks, JHEP 04 (2017) 079 [arXiv: 1609.04783] [INSPIRE].

[43] F.J. Botella and L.-L. Chau, Anticipating the Higher Generations of Quarks from Rephasing Invariance of the Mixing Matrix, Phys. Lett. B 168 (1986) 97 [InSPIRE].

[44] A. de Gouvêa and J. Jenkins, The Physical Range of Majorana Neutrino Mixing Parameters, Phys. Rev. D 78 (2008) 053003 [arXiv:0804.3627] [INSPIRE].

[45] CMS collaboration, Search for vector-like $T$ and $B$ quark pairs in final states with leptons at $\sqrt{s}=13 \mathrm{TeV}, J H E P 08$ (2018) 177 [arXiv: 1805.04758] [INSPIRE].

[46] ATLAS collaboration, Combination of the searches for pair-produced vector-like partners of the third-generation quarks at $\sqrt{s}=13 \mathrm{TeV}$ with the ATLAS detector, Phys. Rev. Lett. 121 (2018) 211801 [arXiv: 1808.02343] [INSPIRE].

[47] CMS collaboration, A search for bottom-type, vector-like quark pair production in a fully hadronic final state in proton-proton collisions at $\sqrt{s}=13 \mathrm{TeV}$, Phys. Rev. D 102 (2020) 112004 [arXiv: 2008.09835] [INSPIRE]. 
[48] Particle Data collaboration, Review of Particle Physics, Prog. Theor. Exp. Phys. 2020 (2020) 083C01 [INSPIRE].

[49] C.-Y. Seng, M. Gorchtein, H.H. Patel and M.J. Ramsey-Musolf, Reduced Hadronic Uncertainty in the Determination of $V_{u d}$, Phys. Rev. Lett. 121 (2018) 241804 [arXiv: 1807.10197] [INSPIRE].

[50] B. Belfatto, R. Beradze and Z. Berezhiani, The CKM unitarity problem: A trace of new physics at the TeV scale?, Eur. Phys. J. C 80 (2020) 149 [arXiv: 1906. 02714] [inSPIRE].

[51] K. Cheung, W.-Y. Keung, C.-T. Lu and P.-Y. Tseng, Vector-like Quark Interpretation for the CKM Unitarity Violation, Excess in Higgs Signal Strength, and Bottom Quark Forward-Backward Asymmetry, JHEP 05 (2020) 117 [arXiv : 2001. 02853] [INSPIRE].

[52] J.A. Aguilar-Saavedra, Effects of mixing with quark singlets, Phys. Rev. D 67 (2003) 035003 [Erratum ibid. 69 (2004) 099901] [hep-ph/0210112] [INSPIRE].

[53] Flavour Lattice Averaging Group, FLAG Review 2019: Flavour Lattice Averaging Group (FLAG), Eur. Phys. J. C 80 (2020) 113 [arXiv:1902.08191] [inSPIRE].

[54] A.J. Buras, M. Jamin and P.H. Weisz, Leading and Next-to-leading QCD Corrections to $\epsilon$ Parameter and $B^{0}-\bar{B}^{0}$ Mixing in the Presence of a Heavy Top Quark, Nucl. Phys. B 347 (1990) 491 [INSPIRE].

[55] J.A. Aguilar-Saavedra, R. Benbrik, S. Heinemeyer and M. Pérez-Victoria, Handbook of vectorlike quarks: Mixing and single production, Phys. Rev. D 88 (2013) 094010 [arXiv: 1306.0572] [INSPIRE].

[56] T. Morozumi, Y. Shimizu, S. Takahashi and H. Umeeda, Effective theory analysis for vector-like quark model, Prog. Theor. Exp. Phys. 2018 (2018) 043B10 [arXiv:1801.05268] [INSPIRE].

[57] A.J. Buras, J. Girrbach, D. Guadagnoli and G. Isidori, On the Standard Model prediction for $\mathcal{B}\left(B_{s, d} \rightarrow \mu^{+} \mu^{-}\right)$, Eur. Phys. J. C 72 (2012) 2172 [arXiv:1208.0934] [InSPIRE].

[58] R. Aleksan, B. Kayser and D. London, Determining the quark mixing matrix from CP-violating asymmetries, Phys. Rev. Lett. 73 (1994) 18 [hep-ph/9403341] [InSPIRE].

[59] A.J. Buras, D. Guadagnoli and G. Isidori, On $\epsilon_{K}$ Beyond Lowest Order in the Operator Product Expansion, Phys. Lett. B $6 \mathbf{6 8}$ (2010) 309 [arXiv: 1002.3612] [INSPIRE].

[60] G. Isidori and R. Unterdorfer, On the short distance constraints from $K_{L, S} \rightarrow \mu^{+} \mu^{-}$, JHEP 01 (2004) 009 [hep-ph/0311084] [INSPIRE].

[61] G. Buchalla and A.J. Buras, The rare decays $K \rightarrow \pi \nu \bar{\nu}, B \rightarrow X \nu \bar{\nu}$ and $B \rightarrow l^{+} l^{-}: A n$ Update, Nucl. Phys. B 548 (1999) 309 [hep-ph/9901288] [InSPIRE].

[62] G. Buchalla and A.J. Buras, QCD corrections to rare $K$ and $B$ decays for arbitrary top quark mass, Nucl. Phys. B 400 (1993) 225 [INSPIRE].

[63] A.J. Buras and L. Silvestrini, Upper bounds on $K \rightarrow \pi \nu \bar{\nu}$ and $K_{L} \rightarrow \pi^{0} e^{+} e^{-}$from $\epsilon^{\prime} / \epsilon$ and $K_{L} \rightarrow \mu^{+} \mu^{-}$, Nucl. Phys. B 546 (1999) 299 [hep-ph/9811471] [InSPIRE].

[64] NA48 collaboration, A Precision measurement of direct CP-violation in the decay of neutral kaons into two pions, Phys. Lett. B 544 (2002) 97 [hep-ex/0208009] [INSPIRE].

[65] KTEV collaboration, Precise Measurements of Direct CP-violation, CPT Symmetry, and Other Parameters in the Neutral Kaon System, Phys. Rev. D 83 (2011) 092001 [arXiv: 1011.0127] [INSPIRE]. 
[66] J. Aebischer, C. Bobeth and A.J. Buras, On the importance of NNLO QCD and isospin-breaking corrections in $\varepsilon^{\prime} / \varepsilon$, Eur. Phys. J. C 80 (2020) 1 [arXiv:1909.05610] [INSPIRE].

[67] J. Aebischer, C. Bobeth and A.J. Buras, $\varepsilon^{\prime} / \varepsilon$ in the Standard Model at the Dawn of the 2020s, Eur. Phys. J. C 80 (2020) 705 [arXiv:2005. 05978] [inSPIRE].

[68] RBC and UKQCD collaborations, Direct CP-violation and the $\Delta I=1 / 2$ rule in $K \rightarrow \pi \pi$ decay from the standard model, Phys. Rev. D 102 (2020) 054509 [arXiv:2004.09440] [INSPIRE].

[69] V. Cirigliano, H. Gisbert, A. Pich and A. Rodríguez-Sánchez, Isospin-violating contributions to $\epsilon^{\prime} / \epsilon$, JHEP 02 (2020) 032 [arXiv:1911.01359] [InSPIRE].

[70] ALEPH, DELPHI, L3, OPAL, SLD collaborations, LEP Electroweak Working Group, SLD Electroweak Group and SLD Heavy Flavour Group, Precision electroweak measurements on the $Z$ resonance, Phys. Rept. 427 (2006) 257 [hep-ex/0509008] [INSPIRE].

[71] J. Haller, A. Hoecker, R. Kogler, K. Mönig, T. Peiffer and J. Stelzer, Update of the global electroweak fit and constraints on two-Higgs-doublet models, Eur. Phys. J. C 78 (2018) 675 [arXiv: 1803.01853] [INSPIRE].

[72] F. Feroz, M.P. Hobson, E. Cameron and A.N. Pettitt, Importance Nested Sampling and the MultiNest Algorithm, Open J. Astrophys. 2 (2019) 10 [arXiv:1306.2144] [INSPIRE].

[73] F. Feroz, M.P. Hobson and M. Bridges, MultiNest: an efficient and robust Bayesian inference tool for cosmology and particle physics, Mon. Not. Roy. Astron. Soc. 398 (2009) 1601 [arXiv: 0809.3437] [INSPIRE].

[74] F. Feroz and M.P. Hobson, Multimodal nested sampling: an efficient and robust alternative to MCMC methods for astronomical data analysis, Mon. Not. Roy. Astron. Soc. 384 (2008) 449 [arXiv: 0704.3704] [INSPIRE].

[75] J. Buchner et al., X-ray spectral modelling of the AGN obscuring region in the CDFS: Bayesian model selection and catalogue, Astron. Astrophys. 564 (2014) A125 [arXiv: 1402.0004] [INSPIRE].

[76] Fermilab Lattice and MILC collaborations, $B_{(s)}^{0}$-mixing matrix elements from lattice QCD for the Standard Model and beyond, Phys. Rev. D 93 (2016) 113016 [arXiv: 1602.03560] [INSPIRE].

[77] P. Marquard, A.V. Smirnov, V.A. Smirnov, M. Steinhauser and D. Wellmann, $\overline{M S}$-on-shell quark mass relation up to four loops in $Q C D$ and a general $\mathrm{SU}(N)$ gauge group, Phys. Rev. D 94 (2016) 074025 [arXiv: 1606.06754] [INSPIRE].

[78] S. Antusch and V. Maurer, Running quark and lepton parameters at various scales, JHEP 11 (2013) 115 [arXiv:1306.6879] [InSPIRE].

[79] T. Jubb, M. Kirk, A. Lenz and G. Tetlalmatzi-Xolocotzi, On the ultimate precision of meson mixing observables, Nucl. Phys. B 915 (2017) 431 [arXiv:1603.07770] [InSPIRE].

[80] M. Artuso, G. Borissov and A. Lenz, CP violation in the $B_{s}^{0}$ system, Rev. Mod. Phys. 88 (2016) 045002 [Addendum ibid. 91 (2019) 049901] [arXiv:1511.09466] [INSPIRE].

[81] J. Brod and M. Gorbahn, Next-to-Next-to-Leading-Order Charm-Quark Contribution to the CP Violation Parameter $\epsilon_{K}$ and $\Delta M_{K}$, Phys. Rev. Lett. 108 (2012) 121801 [arXiv:1108.2036] [INSPIRE]. 
[82] J. Brod, M. Gorbahn and E. Stamou, Standard-Model Prediction of $\epsilon_{K}$ with Manifest Quark-Mixing Unitarity, Phys. Rev. Lett. 125 (2020) 171803 [arXiv:1911.06822] [INSPIRE].

[83] K. Ishiwata, Z. Ligeti and M.B. Wise, New Vector-Like Fermions and Flavor Physics, JHEP 10 (2015) 027 [arXiv: 1506.03484] [INSPIRE].

[84] ATLAS collaboration, Measurement of the CP-violation phase $\phi_{s}$ in $B_{s} \rightarrow J / \psi \phi$ decays in ATLAS at $13 \mathrm{TeV}$, ATLAS-CONF-2019-009 (2019).

[85] LHCb collaboration, Updated measurement of time-dependent CP-violating observables in $B_{s}^{0} \rightarrow J / \psi K^{+} K^{-}$decays, Eur. Phys. J. C 79 (2019) 706 [Erratum ibid. 80 (2020) 601] [arXiv: 1906.08356] [INSPIRE].

[86] LHCb collaboration, Measurement of the CKM angle $\gamma$ using $B^{0} \rightarrow D K^{* 0}$ with $D \rightarrow K_{S}^{0} \pi^{+} \pi^{-}$decays, JHEP 08 (2016) 137 [arXiv: 1605.01082] [INSPIRE].

[87] LHCb collaboration, Physics case for an LHCb Upgrade II - Opportunities in flavour physics, and beyond, in the HL-LHC era, arXiv:1808.08865 [INSPIRE].

[88] T. Inami and C.S. Lim, Effects of Superheavy Quarks and Leptons in Low-Energy Weak Processes $k_{L} \rightarrow \mu \bar{\mu}, K^{+} \rightarrow \pi^{+} \nu \bar{\nu}$ and $K_{0} \rightarrow \bar{K}_{0}$, Prog. Theor. Phys. 65 (1981) 297 [Erratum ibid. 65 (1981) 1772] [INSPIRE]. 